\title{
Global Studies of the Host-Parasite Relationships between Ectoparasitic Mites of the Family Syringophilidae and Birds of the Order Columbiformes
}

\author{
Katarzyna Kaszewska-Gilas ${ }^{1, *(\mathbb{D})}$, Jakub Ziemowit Kosicki ${ }^{2}{ }^{D}$, Martin Hromada $^{3,4}$ (D) and Maciej Skoracki $^{1,3}{ }^{(D)}$ \\ 1 Department of Animal Morphology, Faculty of Biology, Adam Mickiewicz University, 61-614 Poznań, Poland; \\ skoracki@amu.edu.pl \\ 2 Department of Avian Biology and Ecology, Faculty of Biology, Adam Mickiewicz University, \\ 61-614 Poznań, Poland; kubako@amu.edu.pl \\ 3 Laboratory and Museum of Evolutionary Ecology, Department of Ecology, Faculty of Humanities and \\ Natural Sciences, University of Presov, 08001 Prešov, Slovakia; hromada.martin@gmail.com \\ 4 Faculty of Biological Sciences, University of Zielona Góra, 65-516 Zielona Góra, Poland \\ * Correspondence: k.kaszewska@amu.edu.pl
}

check for updates

Citation: Kaszewska-Gilas, K.; Kosicki, J.Z.; Hromada, M.; Skoracki, M. Global Studies of the Host-Parasite Relationships between Ectoparasitic Mites of the Family Syringophilidae and Birds of the Order Columbiformes. Animals 2021, 11, 3392. https://doi.org/10.3390/ ani11123392

Academic Editors: Joanna N. Izdebska and Leszek Rolbiecki

Received: 31 October 2021

Accepted: 23 November 2021

Published: 27 November 2021

Publisher's Note: MDPI stays neutral with regard to jurisdictional claims in published maps and institutional affiliations.

Copyright: (c) 2021 by the authors. Licensee MDPI, Basel, Switzerland. This article is an open access article distributed under the terms and conditions of the Creative Commons Attribution (CC BY) license (https:// creativecommons.org/licenses/by/ $4.0 /)$.
Simple Summary: Mites of the family Syringophilidae (Acariformes: Cheyletoidea)—also called quill mites-are permanent and highly specialized ectoparasites of birds living inside the calamus of the various types of the feathers. In the present paper, we conducted a study focused on prevalence, host specificity, networks, and phylogeny of the syringophilid mites parasitizing on pigeon and doves (Columbiformes). We postulate that the Syringophilidae mites and Columbiformes bird system represent a model which can be used in a broader study of the relationship between hosts and parasites.

\begin{abstract}
The quill mites belonging to the family Syringophilidae (Acari: Prostigmata: Cheyletoidea) are obligate ectoparasites of birds. They inhabit different types of the quills, where they spend their whole life cycle. In this paper, we conducted a global study of syringophilid mites associated with columbiform birds. We examined 772 pigeon and dove individuals belonging to 112 species (35\% world fauna) from all zoogeographical regions (except Madagascan) where Columbiformes occur. We measured the prevalence (IP) and the confidence interval (CI) for all infested host species. IP ranges between 4.2 and 66.7 (CI 0.2-100). We applied a bipartite analysis to determine host-parasite interaction, network indices, and host specificity on species and whole network levels. The Syringophilidae-Columbiformes network was composed of 25 mite species and 65 host species. The bipartite network was characterized by a high network level specialization $\mathrm{H} 2^{\prime}=0.93$, high nestedness $\mathrm{N}=0.908$, connectance $\mathrm{C}=0.90$, and high modularity $\mathrm{Q}=0.83$, with 20 modules. Moreover, we reconstructed the phylogeny of the quill mites associated with columbiform birds on the generic level. Analysis shows two distinct clades: Meitingsunes + Psittaciphilus, and Peristerophila + Terratosyringophilus.
\end{abstract}

Keywords: Acari; biodiversity; quill mites; pigeons and doves; network

\section{Introduction}

Knowing how many species inhabit Earth is among the most fundamental questions in science [1]. Despite often being neglected [2], one of the major components of biodiversity are parasites [3], comprising at least half of all species [4-6]; up to 75\% of all interactions in food webs involve a parasitic species [3]. Many estimates of global species diversity of parasites are based on extrapolations of patterns of host specificity [2]; however, a contrast between the proportion that parasites comprise in local and global faunas suggests that parasites are most probably less host specific and more widespread than local scale studies suggest [6]. To get over such difficulties, it has been increasingly recognized that biotic 
interactions matter not only at local but also at regional, continental, or global spatial scales [7]. Thus, in determining the host specificity of parasites, more precise data on different parasitic taxa on different scales, from local to global, are crucially needed.

The networks can be useful to illustrate and analyze relationships and ecological interaction between any type of community [8]. Such analyses not only give a visual graph links between two trophic levels, but they also offer an opportunity to quantify indices such as parasite-host specificity and species richness in hosts, and they give a topological description of connectance, nestedness, or modularity [8,9].

The monophyletic Columbiformes are one of the oldest and the most diverse nonpasserine clade of Neoaves, comprising more than 320 species grouped in one family Columbidae [10]. Pigeons and doves can be found in all zoogeographical regions except the high Arctic and Antarctic and adjacent islands [11]. Despite some studies suggesting as old as Cretaceous origin of Columbiformes [12], there is a major consensus that they diverged from other basal land bird clades in Eocene [13,14]; radiation of major extant lineages continued until the Miocene [15]. Thus, this group can serve as a good model for global studies of their ectoparasite richness, interactions, and specificity.

The quill mites belonging to the family Syringophilidae (Acariformes: Prostigmata) are highly specialized parasites of birds [16] that live inside the quills of feathers. These obligatory ectoparasites pierce the quill wall with extremely long stylet-like movable digits of chelicerae. Each stylet can be extended independently from the other, this movement occurs in the course of piercing the wall of quill and thus feed on live tissue fluids of their avian hosts [16-19].

Currently, the fauna of Syringophilidae comprises about 400 species belonging to 63 genera and associated with 27 bird orders [20]. However, Johnson and Kethley suggested that, considering their potential host richness, the number of quill mite species can reach as much as 5000 [21]. Most of the quill mites are either restricted to only one host species (monoxenous parasites) or adjusted to live in closely related hosts (oligoxenous parasites) $[22,23]$. However, the host specificity of syringophilids is still insufficiently investigated.

Until now, trophic interaction analyses and bipartite networks have been used for the description of quill mite associations with the following host groups: sunbirds (Passeriformes: Nectariniidae) [24] estrildids (Estrildidae) [25], and doves and pigeons (Columbiformes: Columbidae) [26]. However, more detailed studies of network indices such as connectance, modularity, nestedness and nest specificity of the quill mites from family Syringophilidae are still needed. The analysis of bipartite network metrics obtained for global quill mite-columbiform trophic interactions can shed more light on the architecture and relation between host and parasites.

Quill mite fauna associated with the members of Columbiformes comprises 25 quill mite species belonging to the following genera: Meitingsunes Glowska \& Skoracki 2010, (8 species), Peristerophila Kethley, 1970 (6 species), Psittaciphilus Fain, Bochkov \& Mironov 2000 (2 species), Terratosyrinophilus Bochkov \& Pérez 2002, (2 species), and Gunabopicobia Skoracki \& Hromada, 2013 (7 species), recorded from 65 bird species belonging to 22 pigeons and doves genera. The quill mites-columbiform fauna was studied by Hirst [27], Clark [28], Kethley [16], Lawrence [29], Casto [30,31], Bochkov and Mironov [32], Fain et al. [33], Bochkov and Perez [34], Bochkov and Fain [35], Bochkov et al. [36], Skoracki and Glowska [37], Nattres and Skoracki [38], Glowska and Skoracki [39], Skoracki [22], Skoracki and Dabert [40], Skoracki and Hromada [41], Kaszewska and Skoracki [42], and Kaszewska et al. [43-46].

Historical Review of Quill Mite Genera Associated with Doves and Pigeons

Among five genera of the quill mites infested birds of the order Columbiformes, only one-Gunabopicobia—belongs to the subfamily Picobiinae. The first species of this subfamily-Syringophilus zumpti-was described by Lawrence in 1959 based on the material collected from Streptopelia capicola (type host) [29]. In 1970, Kethley moved this species 
to the genus Picobia in the subfamily Picobiinae. In 2011, Skoracki established a new genus Neopicobia and placed P. zumpti in its species content [22]. However, two years later, considering morphological details (hypostomal apex bumpy and apodemes I without thorn-like protuberances), it was moved by Skoracki and Hromada [41] a new monotypic genus Gunabopicobia. New host records as well as numerous new Gunabopicobia species were described by Kaszewska et al. [42] and Skoracki et al. [47].

Members of the subfamily Syringophilinae associated with columbiform birds belong to four genera. The revision of Syringophilidae conducted by Kethley in 1970 resulted in a description of a new genus Peristerophila. In 2002, Bochkov and Pérez [34] erected a new genus-Castosyringophilus_closely related to Peristerophila. They also moved P. mucuya Casto, 1980 to Castosyringophilus. Subsequently, new quill mites species (12 species) from both genera with new hosts records belonging to the orders Columbiformes, Accipitriformes, and Falconiformes were given in the following papers: Bochkov and Fain [35], Skoracki et al. [48]; Skoracki [22], Skoracki and Glowska [37]; Kaszewska et al. [43,46]. In 2020, Skoracki et.al. [49] carried out a comparative study of the ontogeny and morphological structures of the quill mites belonging to genus Peristerophila. The results of this study indicated that the females of this genus, which are characterized by the presence of the two morphotypes: homeomorph forms belong to the genus Peristerophila while the heteromorphy ones formerly referred to the genus Castosyringophilus. Therefore, considering the ontogeny, the genus Castosyringophilus was synonymized with the genus Peristerophila. In 2003, Bochkov and Fain [35] published the results of their taxonomic studies on syringophilid mites associated with parrots, and established the genus Terratosyringophilus. They moved the previously described species Peristerophila longisoma Casto, 1979 recorded from Zenaida asiatica to the genus Terratosyringophilus. To date, Terratosyringophilus is comprised of five quill mites species infesting both doves and parrots. The other genus known from doves and parrots is Psittaciphilus, described by Fain et al. in 2000 [33]. Originally, Fain and co-authors recorded it only from parrots, but later, Kaszewska and Skoracki [42] found two new quill mites species of this genus, P. montanus and P. patagioenas, on columbiform birds. The results of this study allowed to addition of another genus to Columbiformes-Psittaciformes hosts group. The last genus associated with columbiforms closely related to Psittaciphilus is Meitingsunes described by Glowska and Skoracki in 2010 [39]. Type species of this genus-M. zenadourae-was originally described as Syringophilus zenadourae by Clark 1964 [28]. In taxonomic revision of syringophilids, Kethley [16], moved this species to the Peristerophila genus. Finally, Glowska and Skoracki 2010 [39] based on morphological difference (apodemes I divergent, not fused to apodemes II) established a new genus-Meitingsunes-for this species. In 2011-2020, numerous taxonomic studies added five new quill mite species associated with birds from order Columbiformes. Currently, only one species of the letter genus, $M$. caprimulgus, has been recorded from another bird order, such as Caprimulgiformes [50].

Recent examples of studies on multispecies interactions at macroscales can be broadly grouped into two analytical approaches: analyses of species richness and ecological networks. The ecological network approach usually relies on observed interactions among multiple species at fine spatial resolutions [7].

Therefore, in this study, we focus on describing the richness, interactions, and measuring the specialization of syringophilid ectoparasites and their columbiform hosts in a global scale. Moreover, we reconstruct the syringophilid phylogeny at the generic level. Additionally, based on an earlier study, we summarize all taxonomic and locality records to create a worldwide distribution of the quill mites associated with birds from the order Columbiformes. We also discuss the host-parasite relationships between syringophilid species and columbiform birds.

\section{Materials and Methods}

In the present study, we re-examined the ornithological collections of the columbiform specimens housed in the Bavarian State Collection of Zoology, Munich, Germany 
(ZSM) and Museum of Natural History, Nairobi, Kenya (NMK). These bird collections have been previously used as donors of mite species described or recorded in the several published papers (see Skoracki and Dabert [40]; Skoracki and Glowska [37]; Glowska and Skoracki [39], Skoracki [22]; Skoracki and Hromada [41]; Skoracki et al. [47]; Kaszewska and Skoracki [42]; Kaszewska et al. [42-46]). We also analyzed the host specimens collected from frozen collections housed in several veterinary centers. Bird specimen was examined using a dissecting microscope and the infested quills were opened with a fine scalpel. From each bird specimen, we removed one wing covert, about 5 under-tail coverts, and about 10 contour feathers. Before mounting, mites were softened and cleared in Nesbitt's solution at room temperature for three days [22], and then mites were mounted on slides in Hoyer's medium.

\subsection{Bipartite Networks and Statistics}

The bipartite graph consists of rectangles representing compartment species and the width is proportion to the sum of interaction involving this species. Interacting species are linked by lines whose width is proportional to the number of interactions [51]. To visualize patterns in the studied host-parasite-ecological web, we used the 'bipartite' package available for R software [51]. To visualize bipartite networks we used functions plotweb (Figure 1) and visweb (Figure S1). For all host species recorded in earlier papers without information about prevalence, we gave score 1. Indices were calculated by using networklevel and network-species functions available in bipartite packages.

We calculated the following bipartite index: network specialization $\left(\mathrm{H}^{\prime}\right)$ nestedness $(\mathrm{N})$, connectance $(\mathrm{C})$, and modularity $(\mathrm{Q})$, to measured interaction on species-level we used species specialization metrics $\left(\mathrm{d}^{\prime}\right)$. For this purpose, we prepared the matrices where quill mites species are in the rows (parasites) and the bird species (host) in the columns. ' $\mathrm{H} 2$ ' network-level measure of specialization, based on the deviation of a species' realized number of interactions and that expected from each species' total number of interactions [52]. Values of $\mathrm{H}_{2}$ ' range from 0 to 1 , where 0 indicates low specialization, while 1 suggests high specialization [53]. We also calculated the connectance, defined as the proportion of possible links observed in the network [54], ranged from 0 (low connectance in the network) to 1 to imply more connectance in the network. Nestedness measures how many interactions realized by specialists are a subset of those realized by generalists. The base metric of nestedness is the nestedness temperature $\mathrm{T}\left(0^{\circ}-100^{\circ}\right)$, which measures the departure from a perfectly nested interaction matrix [55]. For this study, we used a binary system, where metrics define as $\mathrm{N}=(100-\mathrm{T}) / 100$, with values ranging from 0 to 1 (maximum nestedness) [56-58].To calculate network modularity we calculate 'likelihood' implemented in computeModules in the bipartite library for R; this index is the same value as Q (or M), the modularity as given by Newman [59] or Guimerà \& Amaral [60] and well known from QuanBiMo (Q) library [61], currently not supported. According to network permutation, we obtained $100 \mathrm{Q}$ values (observed likelihood) [62] and compared them with $100 \mathrm{Q}$ values coming from permutations for null models (null likelihood). To test a significant difference between the Q observed and Qnull values, we calculated the null.t.test $(p<0.05)$. For each quill mites species associated with doves and pigeons in the network, we calculated d' index measured specialization at species level [52]. 


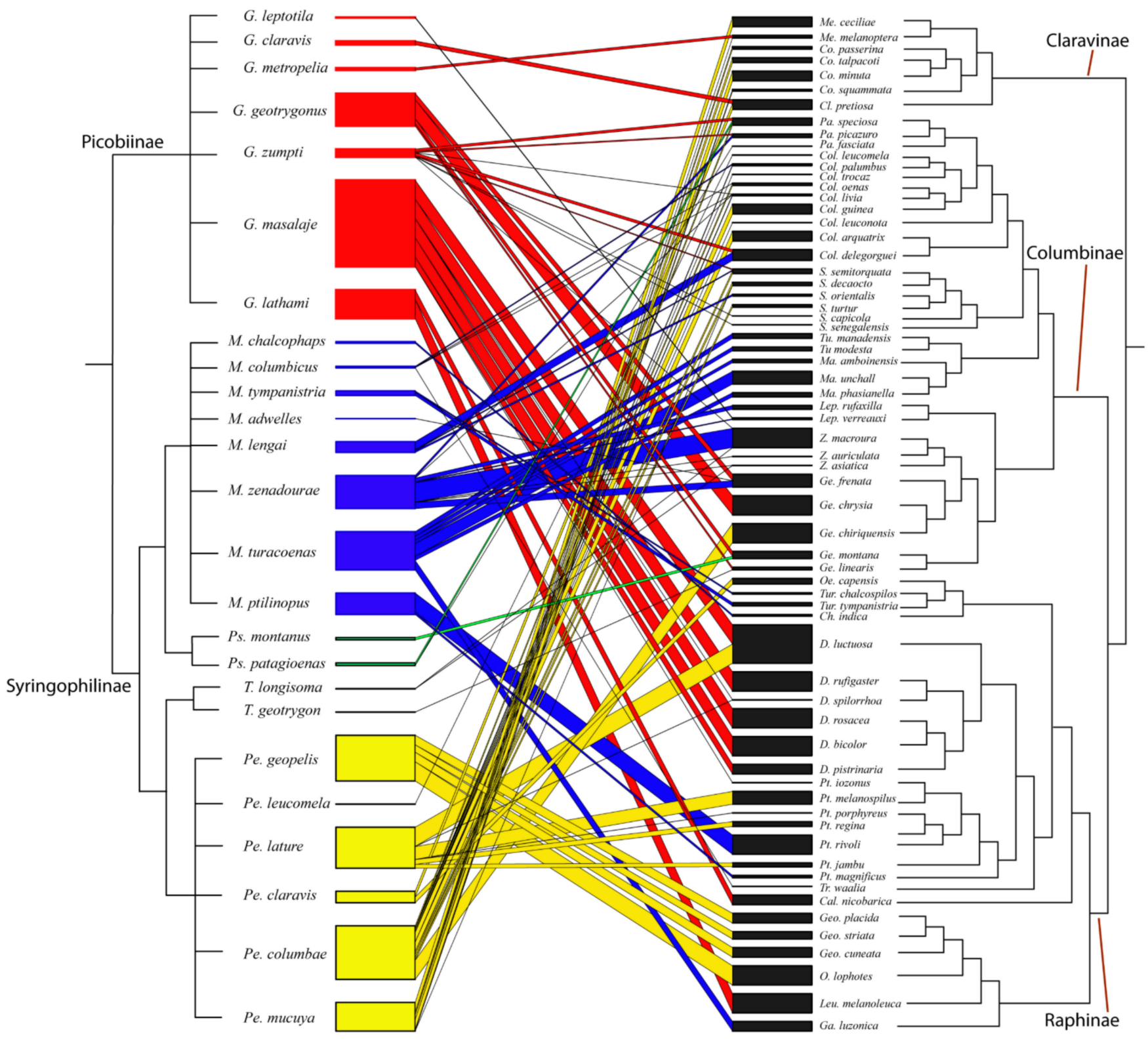

Figure 1. Bipartite network graph of interactions between quill mite species (left) and their doves and pigeons hosts (right).

\subsection{Prevalence}

Descriptive statistics were computed using Quantitative Parasitology on the Web [63], with $95 \%$ confidence intervals (Sterne method).

\subsection{Mite Phylogeny}

In the cladistic analysis, we examined relationships at the generic level. All operational taxonomic units (OTUs) were represented by taxonomic species, i.e., type species for each genus. A free-living predator Cheyletus eruditus (Schrank) and quill-inhabiting predator Metacheletoides numidae Fain both belonging to the sister family Cheyletidae, were used as outgroups in the analyses. Because each particular syringophilid genus is represented by a single species in the present analysis, the character states appearing as autapomorphies represent true synapomorphies for genera.

A total of five OTUs representing all genera associated with columbiform birds, two taxa as the outgroup, and 26 non-additive and unordered morphological characters were included in our data matrix (data matrix and morphological characters are supple- 
mented (Figures S2 and S3)). A detailed discussion of the morphological characters used in the present study is provided by Skoracki [22]; Skoracki et al. [47]. The matrix was done using NEXUS Data Editor 0.5.0 [64]. Analyses of character distribution on the tree were performed in WINCLADA [65]. Only unordered, qualitative, and unweighted characters were used in analyses. We applied a multistate contingent coding strategy [66], which is considered as the most useful among available approaches [67]. Following this strategy, characters with multiple states were interpreted as unordered and not modified into binary characters. Reconstruction of phylogenetic relationships was performed with PAUP 4.0 beta version for IBM [68] in conjunction with PRAP2 [69] to conduct a ratchet analysis (1000 iterations; 10 random cycles, collapsed zero-branches in effect; options are the default). Nodal support was evaluated by Bremer indices calculated with PRAP2. Analysis of character distributions, drawing, and editing of the trees was performed in TreeView 1.5.2. [70].

\subsection{Visualization of Host Phylogeny}

To visualize host phylogeny, a tree of the columbiform species was constructed based on a consensus avian phylogenetic tool available at http://birdtree.org/ (accessed on 5 March 2019) [71]. As the source of our consensus tree, we used the 'Hackett All Species tree' with 1000 randomly generated trees. The most credible tree was then determined using the tool TreeAnnotatorv1.8.2 in the software BEAST v1.8.2 [72]. The consensus tree was then graphically adjusted in FigTree v1.4.2 (Andrew Rambaut, University of Edinburgh, UK; http:/ / tree.bio.ed.ac.uk/software/figtree/ (accessed on 5 March 2019)).

\subsection{Host Specificity}

Host specificity for particular mite species follows Caira et al. [73] and Skoracki et al. [47]. The division stands out monoxenous species (parasite infest single host species), oligoxenous (more than one host, but restricted to one genus), mesostenoxenous (more than one genus of hosts, but restricted to one subfamily), metastenoxenous (more than one subfamily of hosts but restricted to one order), and polyxenous species (more than one order). The common and scientific names of the birds follow Clements et al. [10]. Zoogeographic regions follow Holt et al. [74].

\section{Results}

A total of 772 individuals of pigeons and doves and belonging to 29 genera and 112 species were examined for the presence of quill mites belonging to the family Syringophilidae. Among them, 117 individuals representing 65 species had been infested by the quill mites belonging to the following genera Meitingsunes Glowska \& Skoracki, 2010 (7 species), Peristerophila Kethley 1970 (6), Psittaciphilus Bochkov \& Mironov, 2000 (2), (subfamily Syringophilinae), and Gunabopicobia Bochkov \& Perez, 2002 (7) (subfamily Picobiinae) (Tables 1 and S1).

In total, 22 out of 25 known quill mites species associated with Columbiformes birds were identified (Terratosyringophilus geotrygonus, T. longisoma, and M. adwelles were not found). Among non-infested columbid specimens, some taxa were examined for the presence of quill mites for the first time, for example: Reinwardtoena reinwardtsi, Gymnophaps albertisii, Henicophaps albifrons, and Henicophaps foersteri. 
Table 1. Quill mite species of the family Syringophilidae parasitizing birds of the order Columbiformes with their distribution.

\begin{tabular}{|c|c|c|c|c|}
\hline Quill Mite Species & Host Species & Host Subfamily & Distribution & References \\
\hline \multicolumn{5}{|c|}{ Subfamily Syringophilinae Lavoipierre, 1953} \\
\hline \multicolumn{5}{|c|}{ Genus Meitingsunes Glowska \& Skoracki, 2010} \\
\hline $\begin{array}{l}\text { M. aldwelles Glowska \& } \\
\text { Skoracki, } 2010\end{array}$ & Geotrygon frenata * (Tschudi) & Columbinae & Neot. (Colombia) & {$[39]$} \\
\hline M. columbicus Skoracki, & Columba oenas * Linnaeus & Columbinae & Pala. (Kazakhstan) & [22] \\
\hline “ & Columba livia Gmelin & Columbinae & Pala. (Poland, Slovakia) & {$[22,45]$} \\
\hline “ & Columba palumbus Linnaeus & Columbinae & $\begin{array}{l}\text { Pala. (Germany, } \\
\text { Russia) }\end{array}$ & {$[22,45]$} \\
\hline " & Treron waalia (Meyer) & Raphinae & Afro. (Cameroon) & [43] \\
\hline M. chalcophas & & & Orie. (Indonesia: & \\
\hline $\begin{array}{l}\text { Kaszewska, Skoracki \& } \\
\text { Kavetska, } 2016\end{array}$ & Chalcophas indica* (Linnaeus) & Raphinae & $\begin{array}{l}\text { Timor) } \\
\text { Aust. (Australia) }\end{array}$ & {$[44,45]$} \\
\hline $\begin{array}{c}\text { M. ptilinopus } \\
\text { Kaszewska, Skoracki \& } \\
\text { Hromada, } 2020\end{array}$ & $\begin{array}{c}\text { Ptilinopus magnificus * } \\
\text { Temminck }\end{array}$ & Raphinae & Aust. (Australia) & [45] \\
\hline " & Ptilinopus rivoli (Prevost) & Raphinae & $\begin{array}{l}\text { Ocea. (Papua New } \\
\text { Guinea) }\end{array}$ & [45] \\
\hline $\begin{array}{l}\text { M. lengai Kaszewska, } \\
\text { Skoracki \& Hromada, } \\
2020\end{array}$ & $\begin{array}{c}\text { Columba delegorguei * } \\
\text { Delegorgue }\end{array}$ & Columbinae & Afro. (Tanzania) & [45] \\
\hline “ & Streptopelia orientalis (Latham) & Columbinae & Afro. (Tanzania) & [45] \\
\hline “ & $\begin{array}{l}\text { Streptopelia semitorquata } \\
\text { Ruppell }\end{array}$ & Columbinae & $\begin{array}{l}\text { Pala. (Kazakhstan, } \\
\text { Kyrgyzstan) }\end{array}$ & {$[45]$} \\
\hline $\begin{array}{c}\text { M. turacoenas } \\
\text { Kaszewska, Skoracki \& } \\
\text { Kavetska, } 2016\end{array}$ & Gallicolumba luzonica Scopoli & Raphinae & Orie. (Philippines) & {$[45]$} \\
\hline " & $\begin{array}{l}\text { Macropygia amboinensis } \\
\text { (Linnaeus) }\end{array}$ & Columbinae & $\begin{array}{c}\text { Ocea. (Papua New } \\
\text { Guinea) }\end{array}$ & [45] \\
\hline “ & $\begin{array}{l}\text { Macropygia phasianella } \\
\text { (Temminck) }\end{array}$ & Columbinae & $\begin{array}{l}\text { Orie. (Philippines, } \\
\text { Indonesia: Java) }\end{array}$ & [45] \\
\hline " & Macropygia unchall (Wagler) & Columbinae & $\begin{array}{l}\text { Ocea. (Papua New } \\
\text { Guinea) }\end{array}$ & {$[45]$} \\
\hline " & $\begin{array}{c}\text { Turacoena manadensis * (Quoy } \\
\text { \&Gaimard) }\end{array}$ & Columbinae & $\begin{array}{l}\text { Orie. (Indonesia: } \\
\text { Sulavesi, Nepal) }\end{array}$ & [44] \\
\hline “ & Turacoena modesta (Temminck) & Columbinae & Orie. (Indonesia) & [44] \\
\hline $\begin{array}{c}\text { M. tympanistria } \\
\text { (Skoracki \& Dabert, } \\
\text { 2012) }\end{array}$ & Turtur chalcospilos (Wagler) & Raphinae & Afro. (Tanzania) & {$[40]$} \\
\hline “ & $\begin{array}{l}\text { Turtur tympanistria * } \\
\text { (Temminck) }\end{array}$ & Raphinae & Afro. (Togo, Tanzania) & {$[40,45]$} \\
\hline $\begin{array}{c}\text { M. zenadourae (Clark, } \\
\text { 1964) }\end{array}$ & Columba livia Gmelin & Columbinae & $\begin{array}{l}\text { Near. (USA: Texas); } \\
\text { Afro. (N. Africa, } \\
\text { Djibouti) }\end{array}$ & {$[30,39,45]$} \\
\hline " & Geotrygon frenata (Tschudi) & Columbinae & Neot. (Colombia) & [45] \\
\hline “ & $\begin{array}{c}\text { Leptotila rufaxilla (Richard, } \\
\text { Bernard) }\end{array}$ & Columbinae & $\begin{array}{l}\text { Neot. (Surinam, } \\
\text { Argentina) }\end{array}$ & [45] \\
\hline " & Leptotila verreauxi (Bonaparte) & Columbinae & Neot. (Colombia) & [45] \\
\hline " & Patagioenas picazuro Temminck & Columbinae & Neot. (Paraguay) & [45] \\
\hline " & Zenaida asiatica (Linnaeus) & Columbinae & Near. (USA: Texas) & [75] \\
\hline " & Zenaida auriculata (Murs) & Columbinae & Neot. (Argentina) & [76] \\
\hline " & Zenaida macroura * (Linnaeus) & Columbinae & $\begin{array}{l}\text { Near. (USA: Maryland, } \\
\text { Arizona, San Francisco) }\end{array}$ & {$[28,39,45]$} \\
\hline
\end{tabular}


Table 1. Cont.

\begin{tabular}{|c|c|c|c|c|}
\hline Quill Mite Species & Host Species & Host Subfamily & Distribution & References \\
\hline \multicolumn{5}{|c|}{ Genus Peristerophila Kethley, 1970} \\
\hline P. columbae (Hirst, 1920) & Columba arguatrix (Temminck) & Columbinae & Afro. (Kenya) & [46] \\
\hline " & Columba guinea Linnaeus & Columbinae & $\begin{array}{l}\text { Afro. (S Africa, } \\
\text { Tanzania) }\end{array}$ & [46] \\
\hline " & Columba leuconota (Vigors) & Columbinae & Orie. (Nepal) & [46] \\
\hline " & Columba livia * Gmelin & Columbinae & $\begin{array}{l}\text { Pala. (England, } \\
\text { Macedonia, Poland, } \\
\text { Turkey); Near. (Canada, } \\
\text { USA); Orie. (India); } \\
\text { Sa-Arab. (Iran) }\end{array}$ & {$[22,27,32,38,46]$} \\
\hline “ & Columba oenas Linnaeus & Columbinae & Pala. (Germany) & [46] \\
\hline “ & Columba palumbus Linnaeus & Columbinae & $\begin{array}{l}\text { Pala. (Germany, } \\
\text { England) }\end{array}$ & {$[46]$} \\
\hline " & Columba trocaz Heineken & Columbinae & Pala. (Portugal) & {$[46]$} \\
\hline " & Geotrygon chiriquensis Sclater & Columbinae & Pana. (Panama) & [46] \\
\hline “ & Patagioenas speciosa (Gmelin) & Columbinae & Neot. (Surinam) & [46] \\
\hline " & Streptopelia capicola (Sundevall) & Columbinae & Afro. (Angola) & [46] \\
\hline “ & $\begin{array}{l}\text { Streptopelia decaocto } \\
\text { (Frivaldszky) }\end{array}$ & Columbinae & Sa-Arab. (Jordan) & [22] \\
\hline " & $\begin{array}{c}\text { Streptopelia decipiens (Hartlaub } \\
\text { \& Finsch.) }\end{array}$ & Columbinae & $\begin{array}{l}\text { Pala. (Macedonia), } \\
\text { Afro. (Tanzania) }\end{array}$ & [46] \\
\hline " & Streptopelia orientalis (Latham) & Columbinae & Orie. (Japan) & [46] \\
\hline “ & $\begin{array}{l}\text { Streptopelia semitorquata } \\
\text { (Ruppell) }\end{array}$ & Columbinae & $\begin{array}{c}\text { Afro. (Angola, } \\
\text { Tanzania, D. R. Congo) }\end{array}$ & {$[46]$} \\
\hline “ & $\begin{array}{c}\text { Streptopelia tranquebarica } \\
\text { (Hermann) }\end{array}$ & Columbinae & Orie. (China) & {$[46]$} \\
\hline “ & Streptopelia turtur (Linnaeus) & Columbinae & $\begin{array}{c}\text { Pala. (Germany, Greece, } \\
\text { Hungary, Macedonia) }\end{array}$ & {$[46]$} \\
\hline $\begin{array}{l}\text { P. claravis (Skoracki \& } \\
\text { Glowska, 2008) }\end{array}$ & Claravis pretiosa * Ferrari-Pérez & Claravinae & $\begin{array}{c}\text { Neot. (Bolivia, } \\
\text { Colombia, Paraguay), } \\
\text { Pana. (Panama) }\end{array}$ & {$[37,46]$} \\
\hline " & Oena capensis (Linnaeus) & Raphinae & $\begin{array}{l}\text { Afro. (Ethiopia, Sudan, } \\
\text { Tanzania) }\end{array}$ & {$[46]$} \\
\hline \multirow{2}{*}{$\begin{array}{c}\text { P. geopelis Kaszewska, } \\
\text { Skoracki, Kosicki \& } \\
\text { Hromada, } 2020 \\
\text { “ }\end{array}$} & Geopelia cuneata (Latham) & Raphinae & Austr. (Australia) & [46] \\
\hline & Geopelia placida Gould & Raphinae & Austr. (Australia) & [46] \\
\hline “ & Geopelia striata * (Linnaeus) & Raphinae & $\begin{array}{l}\text { Orie. (Indonesia: } \\
\text { Celebes, Java, Sumatra) }\end{array}$ & [46] \\
\hline “ & Ocyphaps lophotes (Temminck) & Raphinae & Austr. (Australia) & [46] \\
\hline $\begin{array}{c}\text { P. lature Kaszewska, } \\
\text { Kavetska \& Skoracki, } \\
2014\end{array}$ & Ducula luctuosa * (Temminck) & Raphinae & Austr. (Australia) & [43] \\
\hline " & Ducula spilorrhoa (Gray) & Raphinae & $\begin{array}{l}\text { Austr. (Papua New } \\
\text { Guinea) }\end{array}$ & [43] \\
\hline “ & Ptilinopus jambu (Gmelin) & Raphinae & $\begin{array}{l}\text { Orie. (Indonesia: } \\
\text { Sumatra) }\end{array}$ & [43] \\
\hline " & $\begin{array}{l}\text { Ptilinopus melanospilus } \\
\text { (Salvadori) }\end{array}$ & Raphinae & $\begin{array}{l}\text { Orie. (Indonesia: } \\
\text { Mount Gade) }\end{array}$ & [43] \\
\hline “ & $\begin{array}{l}\text { Ptilinopus porphyreus } \\
\text { (Temminck) }\end{array}$ & Raphinae & Orie. (Indonesia: Java) & [43] \\
\hline “ & Ptilinopus regina (Swainson) & Raphinae & $\begin{array}{l}\text { Orie. (Indonesia: } \\
\text { Marina Isl.) }\end{array}$ & [43] \\
\hline $\begin{array}{l}\text { P. leucomela Kaszewska, } \\
\text { Skoracki, Kosicki \& } \\
\text { Hromada, } 2020\end{array}$ & Columba leucomela * Temminck & Columbinae & Austr. (Australia) & [46] \\
\hline P. mucuya Casto, 1980 & Columbina minuta Linnaeus & Claravinae & Neot. (Paraguay) & {$[46]$} \\
\hline
\end{tabular}


Table 1. Cont.

\begin{tabular}{|c|c|c|c|c|}
\hline Quill Mite Species & Host Species & Host Subfamily & Distribution & References \\
\hline " & $\begin{array}{l}\text { Columbina passerina * } \\
\text { (Linnaeus) }\end{array}$ & Claravinae & $\begin{array}{c}\text { Neot. (Colombia, } \\
\text { Surinam); Near. (USA) }\end{array}$ & {$[30,46]$} \\
\hline " & Columbina squammata (Lesson) & Claravinae & $\begin{array}{l}\text { Neot. (Brazil, } \\
\text { Paraguay) }\end{array}$ & {$[35,46]$} \\
\hline " & Columbina talpacoti (Temminck) & Claravinae & $\begin{array}{c}\text { Neot. (Brazil, Surinam, } \\
\text { Trinidad and Tobago); } \\
\text { Pala. (Monaco) }\end{array}$ & {$[37,46]$} \\
\hline “ & Geophaps plumifera Gould & Columbinae & Aust. (Australia) & [35] \\
\hline " & Metriopelia ceciliae (Lesson) & Claravinae & Neot. (Peru) & [46] \\
\hline “ & $\begin{array}{c}\text { Metriopelia melanoptera } \\
\text { (Molina) }\end{array}$ & Claravinae & Neot. (Argentina) & [37] \\
\hline “ & Brotogeris versicolurus ${ }^{* *}$ Muller & Psittacidae & Neot. (Brazil) & [35] \\
\hline " & $\begin{array}{c}\text { Psilopsiagon aymara ** } \\
\text { d'Orbigny }\end{array}$ & Psittacidae & Neot. (S. America) & [35] \\
\hline " & $\begin{array}{c}\text { Trichoglossus haematodus ** } \\
\text { (Linnaeus) }\end{array}$ & Psittaculidae & Ori. (Indonesia) & [35] \\
\hline
\end{tabular}

Genus Psittaciphilus, Bochkov \& Mironov, 2000

\begin{tabular}{|c|c|c|c|c|}
\hline $\begin{array}{l}\text { P. montanus Kaszewska } \\
\text { \& Skoracki, } 2018\end{array}$ & Geotrygon montana* (Linnaeus) & Columbinae & $\begin{array}{l}\text { Neot. (Brazil, Trinidad } \\
\text { and Tobago); Pana. } \\
\text { (Panama) }\end{array}$ & [42] \\
\hline $\begin{array}{c}\text { P. patagioenas } \\
\text { Kaszewska \& Skoracki, } \\
2018\end{array}$ & Patagioenas fasciata * (Say) & Columbinae & Neot. (Colombia) & [42] \\
\hline “ & Patagioenas speciosa (Gmelin) & Columbinae & Neot. (Surinam) & [42] \\
\hline \multicolumn{5}{|c|}{ Genus Terratosyringophilus Bochkov and Perez, 2002} \\
\hline $\begin{array}{l}\text { T. geotrygonus Skoracki } \\
\text { \& Glowska, } 2008\end{array}$ & Geotrygon linearis * (Prévost) & Columbinae & Neot. (Venezuela) & {$[37]$} \\
\hline $\begin{array}{c}\text { T. longisoma (Casto, } \\
\text { 1979) }\end{array}$ & Zenaida asiatica * (Linnaeus) & Columbinae & Near. (USA) & [31] \\
\hline " & Zenaida macroura (Linnaeus) & Columbinae & Near. (USA) & {$[37]$} \\
\hline \multicolumn{5}{|c|}{ Subfamily Picobiinae Johnson \& Kethley, 1973} \\
\hline \multicolumn{5}{|c|}{ Genus Gunabopicobia Skoracki \& Hromada, 2013} \\
\hline $\begin{array}{l}\text { G. claravis Kaszewska, } \\
\text { Skoracki \& Hromada, } \\
2018\end{array}$ & $\begin{array}{c}\text { Claravis pretiosa * } \\
\text { (Ferrari-Perez) }\end{array}$ & Claravinae & Neot. (Colombia) & [26] \\
\hline $\begin{array}{c}\text { G. geotrygoni } \\
\text { Kaszewska, Skoracki \& } \\
\text { Hromada, } 2018\end{array}$ & Geotrygon linearis * (Prevost) & Columbinae & Neot. (Venezuela) & [26] \\
\hline " & Geotrygon chrysia Bonaparte & Columbinae & Ocea. (Martinique) & [26] \\
\hline " & Geotrygon frenata (Tschudi) & Columbinae & Neot. (Colombia) & [26] \\
\hline “ & Geotrygon montana (Linnaeus) & Columbinae & Neot. (Paraguay) & [26] \\
\hline $\begin{array}{c}\text { G. masalaje Kaszewska, } \\
\text { Kavetska \& Skoracki, } \\
2014\end{array}$ & Ducula bicolor (Scopoli) & Raphinae & Orie. (Indonesia) & [43] \\
\hline “ & $\begin{array}{c}\text { Ducula rufigaster (Quoy and } \\
\text { Gaimard) }\end{array}$ & Raphinae & $\begin{array}{l}\text { Ocea. (Papua New } \\
\text { Guinea) }\end{array}$ & [43] \\
\hline " & Ducula rosacea (Temminck) & Raphinae & $\begin{array}{l}\text { Orie. (Indonesia: } \\
\text { Semau Isl.) }\end{array}$ & {$[43]$} \\
\hline " & Ducula pistrinaria Bonaparte & Raphinae & $\begin{array}{l}\text { Ocea. (Papua New } \\
\text { Guinea) }\end{array}$ & [43] \\
\hline " & Ducula spilorrhoa (Gray) & Raphinae & $\begin{array}{l}\text { Orie. (Indonesia: } \\
\text { Semau Isl.) }\end{array}$ & {$[43]$} \\
\hline “ & Ducula luctuosa (Temminck) & Raphinae & $\begin{array}{l}\text { Ocea. (Papua New } \\
\text { Guinea) }\end{array}$ & [43] \\
\hline
\end{tabular}


Table 1. Cont.

\begin{tabular}{|c|c|c|c|c|}
\hline Quill Mite Species & Host Species & Host Subfamily & Distribution & References \\
\hline " & Ptilinopus iozonus * Gray & Raphinae & $\begin{array}{l}\text { Ocea. (Papua New } \\
\text { Guinea) }\end{array}$ & [43] \\
\hline $\begin{array}{c}\text { G. metriopelia } \\
\text { Kaszewska, Skoracki } \\
\text { and Hromada, } 2018\end{array}$ & $\begin{array}{l}\text { Metriopelia melanoptera * } \\
\text { (Molina) }\end{array}$ & Claravinae & Neot. (Argentina) & [26] \\
\hline $\begin{array}{c}\text { G. lathami Kaszewska, } \\
\text { Skoracki and Hromada, } \\
2018\end{array}$ & Leucosarcia melanoleuca * Gould & Raphinae & $\begin{array}{l}\text { Orie. (Indonesia); Ocea. } \\
\text { (Papua New Guinea) }\end{array}$ & [26] \\
\hline " & Caloenas nicobarica (Linnaeus) & Raphinae & $\begin{array}{l}\text { Orie. (Indonesia); Ocea. } \\
\text { (Papua New Guinea) }\end{array}$ & [26] \\
\hline $\begin{array}{l}\text { G. leptotila Kaszewska, } \\
\text { Skoracki \& Hromada, } \\
2018\end{array}$ & Leptotila verreauxi * (Bonaparte) & Columbinae & Neot. (Argentina) & [26] \\
\hline $\begin{array}{c}\text { G. zumpti (Lawrence, } \\
\text { 1959) }\end{array}$ & Columba livia Gmelin & Columbinae & $\begin{array}{l}\text { Near. (USA); } \\
\text { Pala. (Poland) }\end{array}$ & {$[36,47]$} \\
\hline “ & Columba delegorquei Delegorgue & Columbinae & Afro. (Tanzania) & [26] \\
\hline " & $\begin{array}{c}\text { Patagioenas picazuro } \\
\text { (Temminck) }\end{array}$ & Columbinae & Neot. (West Brazil) & [26] \\
\hline " & Patagioenas speciosa Gmelin & Columbinae & Neot. (North Brazil) & [26] \\
\hline “ & $\begin{array}{l}\text { Streptopelia capicola * } \\
\text { (Sundevall) }\end{array}$ & Columbinae & Afro. (South Africa) & [29] \\
\hline " & $\begin{array}{l}\text { Streptopelia semitorquata } \\
\text { (Ruppell) }\end{array}$ & Columbinae & Afro. (Ethiopia) & [26] \\
\hline " & $\begin{array}{l}\text { Streptopelia senegalensis } \\
\text { (Linnaeus) }\end{array}$ & Columbinae & Afro.(South Africa) & [41] \\
\hline " & Zenaida macroura (Linnaeus) & Columbinae & Near. (USA) & [28] \\
\hline
\end{tabular}

Zoogeographical regions: Afro.-Afrotropical, Aust.-Australian, Near.-Nearctic, Neot.-Neotropical, Ocea.-Oceanian, Orie.-Oriental, Pala.-Palaearctic, Pana.—Panamanian, Sa-Arab.-Saharo-Arabian, Si-Jap.-Sino-Jappanese (according to Holt et al. [71]). *-type host: **_host from order Psittaciformes; "- previous species name. Locality established based on the host distribution.

\subsection{Prevalence Index Birds from Order Columbiformes}

The index of prevalence (IP) of host species from Columbiformes order ranges from $4.2 \%$ to $100 \%$ ( $\mathrm{IP}=100$ in 17 cases); however, the confidence intervals were wide and ranged from 0.2 to 100 (Table 2). In our material, 49 host species (239 individuals) were not infested by the syringophilid mites.

(1) IP 1-10\% Chalcophaps indica (8.7\%), Columba livia (8.7\%), Columba palumbus (5\%), Columbina squammata $(6.7 \%)$, Leptotila verreauxi $(4.2 \%)$, Patagioenas picazuro $(6.2 \%)$, Streptopelia orientalis (9.1\%), Streptopelia semitorquata (4.8\%), Turtur chalcospilos $(7 \%)$.

(2) IP 11-20\% Claravis pretiosa (20\%), Columba delegorguei (14.3\%), Columba oenas (11.1\%), Columbina raucana (13.3\%), Geotrygon linearis (12.5\%), Geotrygon montana (12.5\%), Leptotila rufaxilla $(20 \%)$, Macropygia amboinensis $(16.7 \%)$, Metriopelia melanoptera $(12.5 \%)$, Patagioenas picazuro (12.5\%), Patagioenas speciosa (12.5\%), Patagioenas speciosa $(12.5 \%)$, Ptilinopus magnificus (11.8\%), Streptopelia semitorquata (14.3\%), Streptopelia turtur (13.3\%), Turacoena modesta (20), Turtur tympanistria (16.7\%).

(3) IP 21-30\% Columbina talpacoti (25\%), Geotrygon montana (25\%), Macropygia phasianella $(21.4 \%)$, Oena capensis (29.4\%), Turacoena manadensis $(25 \%)$.

(4) IP 31-40\% Geotrygon frenata (33\%), Geopelia striata (38.5\%).

(5) IP 41-50\% Caloenas nicobarica (50\%), Columba arquatrix (50\%), Columba delegorguei $(42 \%)$, Columba guinea (50\%), Columbina minuta (50\%), Gallicolumba luzonica $(50 \%)$, Geopelia cuneata (50\%), Geopelia placida (50\%), Metriopelia ceciliae (50\%).

(6) IP 61-70 Macropygia unchall (66.7\%), Ptilinopus melanospilus (66.7\%).

(7) IP 100\% Ducula bicolor, Geotrygon chrysie, Geotrygon chiriquensis, Leucosarcia melanoleuca, Ocyphaps lophotes, Ptilonopus rauca, Zenaida macroura. 
In Table 2. We excluded the following examined but non-infested bird species: Chalcophaps stephani $[\mathrm{N}=2]$, Claravis mondetoura $[\mathrm{N}=2]$, Columba rupestris $[\mathrm{N}=11]$, Columbina cruziana [N = 2], Ducula aenea [N = 4], Ducula carola [N = 2], Ducula concinna [N =1], Ducula finschii [N = 2], Ducula perspicillata [N =1], Ducula zoeae [N = 7], Gallicolumba rufigula [N =1], Geotrygon violacea $[\mathrm{N}=1]$, Gymnophaps albertisii $[\mathrm{N}=2]$, Henicophaps albifrons $[\mathrm{N}=1]$, Henicophaps foersteri $[\mathrm{N}=1]$, Leptotila cassini $[\mathrm{N}=1]$, Leptotrygon veraguensis $[\mathrm{N}=1]$, Macropygia magna [N = 3], Macropygia ruficeps [N = 4], Otidiphaps nobilis [N = 1], Patagioenas raucana [N = 12], Patagioenas cayennensis [N = 18], Patagioenas oenops [N = 4], Patagioenas subvinacea [N = 3], Ptilinopus bernsteinii [N =1], Ptilinopus cinctus [N = 6], Ptilinopus coronulatus [N = 4], Ptilinopus ornatus [N =1], Ptilinopus porphyreus [N = 3], Ptilinopus pulchellus [N =5], Ptilinopus solomonensis $[\mathrm{N}=1]$, Ptilinopus superbus $[\mathrm{N}=6]$, Reinwardtoena reinwardtii $[\mathrm{N}=2]$, Streptopelia picturata $[\mathrm{N}=1]$, Streptopelia roseogrisea $[\mathrm{N}=3]$, Streptopelia senegalensis $[\mathrm{N}=26]$, Streptopelia tranquebarica $[\mathrm{N}=5]$, Treron bicinctus $[\mathrm{N}=3]$, Treron calva $[\mathrm{N}=7]$, Treron capellei $[\mathrm{N}=3]$, Treron curvirostra $[\mathrm{N}=8]$, Treron delalandii $[\mathrm{N}=20]$, Treron fulvicollis $[\mathrm{N}=4]$, Treron olax $[\mathrm{N}=2]$, Treron pompadora $[\mathrm{N}=7]$, Treron sieboldii $[\mathrm{N}=5]$, Treron sphenurus $[\mathrm{N}=3]$, Treron vernanus $[\mathrm{N}=18]$, Zenaida galapagoensis $[\mathrm{N}=8]$.

Table 2. Host species infested by quill mites with habitat and the index of prevalence (IP) and 95\% confidence interval (Sterne's method).

\begin{tabular}{|c|c|c|c|c|c|c|}
\hline \multicolumn{2}{|c|}{ Host Species } & \multirow{2}{*}{$\frac{\text { Exa. }}{4}$} & \multirow{2}{*}{$\begin{array}{c}\text { Inf. } \\
2\end{array}$} & \multirow{2}{*}{$\frac{\text { IP; CI }}{50(9.8-90.2)}$} & \multirow{2}{*}{$\begin{array}{c}\text { Mite Species } \\
\text { G. lathami }\end{array}$} & \multirow{2}{*}{$\begin{array}{l}\text { Habitat } \\
\text { contour }\end{array}$} \\
\hline Caloenas nicobarica * & Nicobar pigeon & & & & & \\
\hline Chalcophaps indica & Grey-capped Emerald Dove & 23 & 2 & $8.7(1.6-27.8)$ & M. chalcophas & coverts \\
\hline Claravis pretiosa & \multirow[t]{2}{*}{ Blue Ground-dove } & \multirow{2}{*}{10} & 3 & $30(8.7-61.9)$ & P. claravis & coverts \\
\hline “* & & & 2 & $20(3.7-55.3)$ & G. claravis & contour \\
\hline Columba arquatrix & African Olive-pigeon & 4 & 2 & $50(9.8-90.2)$ & P. columbae & under-wings cov. \\
\hline \multirow{2}{*}{$\begin{array}{c}\text { Columba delegorguei } \\
\star *\end{array}$} & \multirow[t]{2}{*}{ Delegorgue's Pigeon } & \multirow[t]{2}{*}{7} & 2 & $42(14.9-77.5)$ & M. lengai & under-tail cov. \\
\hline & & & 1 & $14.3(0.7-55.4)$ & G. zumpti & contour \\
\hline Columba guinea & Speckled Pigeon & 4 & 2 & $50(9.8-90.2)$ & P. columbae & under-wings cov. \\
\hline Columba leucomela & White-headed Pigeon & 1 & 1 & $100(5.0-100)$ & P. leucomela & - \\
\hline Columba leuconota & Snow Pigeon & 1 & 1 & $100(5.0-100)$ & P. columbae & - \\
\hline Columba livia & Rock Pigeon & 20 & 1 & $5(0.3-24.4)$ & P. columbae & contour \\
\hline " & & NA & NA & - & G. zumpti & contour \\
\hline “ & & 1 & 1 & $100(5.0-100)$ & M.zenadourae & covert \\
\hline " & & NA & NA & - & M. columbicus & secondaries \\
\hline \multirow{2}{*}{$\begin{array}{c}\text { Columba oenas } \\
\text { " }\end{array}$} & \multirow[t]{2}{*}{ Stock Dove } & NA & NA & - & M. columbicus & secondaries \\
\hline & & 9 & 1 & $11.1(0.6-44.4)$ & P. columbae & under-wings cov. \\
\hline \multirow{2}{*}{ Columba palumbus } & \multirow[t]{2}{*}{ Common Wood-Pigeon } & 20 & 1 & $5(0.3-24.4)$ & M. columbicus & tail cov. \\
\hline & & 20 & 1 & $5(0.3-24.4)$ & P. columbae & covert \\
\hline Columba trocaz & Madeira laurel Pigeon & 1 & 1 & $100(5.0-100)$ & P. columbae & under wing cov. \\
\hline Columbina minuta & Plain-breasted Ground-Dove & 4 & 2 & $50(9.8-90.2)$ & Р.тисиуа & contour \\
\hline Columbina passerina & Common Ground-Dove & 15 & 2 & $13.3(2.4-39.7)$ & Р.тисиуа & secondaries \\
\hline Columbina talpacoti & Ruddy Ground-Dove & 32 & 8 & $25(12.2-42.3)$ & Р.mисиуа & under-wings cov \\
\hline Columbina squammata & Scaled Dove & 11 & 1 & $9.1(0.5-40.5)$ & Р.тисиуа & tertials \\
\hline Ducula bicolor * & Pied Imperial-Pigeon & 2 & 2 & $100(22.4-100)$ & G. masalaje & contour \\
\hline \multirow{2}{*}{$\underset{\text { Ducula luctuosa * }}{ }$} & \multirow[t]{2}{*}{ Silver-tipped Imperial-Pigeon } & 1 & 1 & $100(22.4-100)$ & G. masalaje & contour \\
\hline & & 1 & 1 & $100(22.4-100)$ & P.lature & covert \\
\hline Ducula pistrinaria * & Island Imperial-Pigeon & 2 & 1 & $50(2.5-97.5)$ & G. masalaje & contour \\
\hline Ducula rosacea * & Pink-headed Imperial-Pigeon & 1 & 1 & $100(22.4-100)$ & G. masalaje & contour \\
\hline Ducula rufigaster * & Purple-tailed Imperial-Pigeon & 1 & 1 & $100(22.4-100)$ & G. masalaje & contour \\
\hline \multirow{2}{*}{$\underset{\text { Ducula spilorrhoa * }}{ }$} & \multirow[t]{2}{*}{ Torresian Imperial-Pigeon } & 1 & 1 & $100(5.0-100)$ & G. masalaje & contour \\
\hline & & 1 & 1 & $100(5.0-100)$ & P.lature & - \\
\hline Gallicolumba luzonica & Luzon Bleeding-heart & 2 & 1 & $50(2.5-97.5)$ & M. turacoenas & contour \\
\hline Geopelia cuneata & Diamond Dove & 2 & 1 & $50(2.5-97.5)$ & P. geopelis & covert \\
\hline Geopelia placida & Peaceful Dove & 2 & 1 & $50(2.5-97.5)$ & P. geopelis & contour \\
\hline Geopelia striata & Zebra Dove & 13 & 5 & $38.5(16.6-65.8)$ & P. geopelis & covert \\
\hline Geotrygon chrysie* & Key West Quail-Dove & 1 & 1 & $100(22.4-100)$ & G. geotrygoni & contour \\
\hline
\end{tabular}


Table 2. Cont.

\begin{tabular}{|c|c|c|c|c|c|c|}
\hline \multicolumn{2}{|c|}{ Host Species } & \multirow{2}{*}{$\begin{array}{c}\text { Exa. } \\
1\end{array}$} & \multirow{2}{*}{$\begin{array}{c}\text { Inf. } \\
1\end{array}$} & \multirow{2}{*}{$\begin{array}{c}\text { IP; CI } \\
100(5-100)\end{array}$} & \multirow{2}{*}{$\begin{array}{c}\text { Mite Species } \\
\text { P. columbae }\end{array}$} & \multirow{2}{*}{$\begin{array}{c}\text { Habitat } \\
\text { under-tail cov. }\end{array}$} \\
\hline Geotrygon chiriquensis & Chiriqui Quail-Dove & & & & & \\
\hline \multirow{2}{*}{ Geotrygon frenata } & \multirow[t]{2}{*}{ White-throated Quail-Dove } & \multirow[b]{2}{*}{3} & 1 & $33(1.7-86.5)$ & M. zenadourae & - \\
\hline & & & 1 & $33(1.7-86.5)$ & G. geotrygoni & contour \\
\hline \multirow[t]{2}{*}{ Geotrygon linearis * } & \multirow[t]{2}{*}{ Lined Quail-Dove } & \multirow[t]{2}{*}{8} & 1 & $12.5(0.6-50)$ & G. geotrygoni & contour \\
\hline & & & 1 & $12.5(0.6-50)$ & T. geotrygonus & primaries \\
\hline \multirow{2}{*}{ Geotrygon montana * } & \multirow[t]{2}{*}{ Ruddy Quail-Dove } & \multirow{2}{*}{8} & 2 & $25(4.6-63.5)$ & G. geotrygoni & contour \\
\hline & & & 1 & $12.5(0.6-50)$ & P. montanus & under tail cov. \\
\hline \multirow{2}{*}{$\begin{array}{l}\text { Leptotila verreauxi } \\
\text { "* }\end{array}$} & \multirow[t]{2}{*}{ White-tipped dove } & \multirow{2}{*}{24} & 1 & $4.2(0.2-20.4)$ & M. zenadourae & under-tail cov. \\
\hline & & & 1 & $4.2(0.2-20.4)$ & G. leptotila & contour \\
\hline Leptotila rufaxilla & Gray-fronted Dove & 10 & 2 & $20(3.7-55.3)$ & M. zenadourae & under-tail cov. \\
\hline $\begin{array}{l}\text { Leucosarcia } \\
\text { melanoleuca }\end{array}$ & Wonga Pigeon & 1 & 1 & $100(5-100)$ & G. lathami & contour \\
\hline $\begin{array}{l}\text { Macropygia } \\
\text { amboinensis }\end{array}$ & Amboyna Cuckoo-Dove & 6 & 1 & $16.7(0.9-58.9)$ & M. turacoenas & - \\
\hline Macropygia phasianella & Brown Cuckoo-Dove & 14 & 3 & $21.4(6.1-50)$ & M. turacoenas & $\begin{array}{c}\text { under and } \\
\text { upper-tail cov. }\end{array}$ \\
\hline Macropygia unchall & Barred Cuckoo-Dove & 3 & 2 & $66.7(13.5-98.3)$ & M. turacoenas & under-tail cov. \\
\hline Metriopelia ceciliae & Bare-faced Ground-Dove & 2 & 1 & $50(2.5-97.5)$ & P. muсuya & secondaries, covert \\
\hline $\begin{array}{l}\text { Metriopelia } \\
\text { melanoptera }\end{array}$ & Black-winged Ground-Dove & 8 & 1 & $12.5(0.6-50)$ & G. metriopelia & contour \\
\hline Ocyphaps lophotes & Crested Pigeon & 1 & 1 & $100(5-100)$ & P. geopelis & $\begin{array}{l}\text { small covert } \\
\text { under-tail cov. }\end{array}$ \\
\hline Oena capensis & Namaqua Dove & 17 & 5 & $29.4(12.4-54.4)$ & P. claravis & under-tail cov. \\
\hline Patagioenas fasciata & Band-tailed pigeon & 1 & 1 & $100(5.0-100)$ & P. patagioenas & upper-tail cov. \\
\hline Patagioenas picazuro & Picazuro Pigeon & & 2 & $12.5(2.3-37.2)$ & M. zenadourae & under-wing cov. \\
\hline " $*$ & & 16 & 1 & $6.2(0.3-30.5)$ & G. zumpti & contour \\
\hline Patagioenas speciosa & Scaled Pigeon & & 1 & $12.5(0.6-50)$ & P. columbae & - \\
\hline " * & & 8 & 1 & $12.5(0.6-50)$ & G. zumpti & contour \\
\hline$"$ & & & 1 & $12.5(0.6-50)$ & P. patagioenas & coverts \\
\hline Ptilinopus iozonus * & Orange-Bellied Fruit Dove & 4 & 1 & $25(1.3-75.1)$ & G. masalaje & contour \\
\hline Ptilinopus jambu & Jambu Fruit-Dove & 5 & 1 & $20(1-65,7)$ & P. lature & coverts \\
\hline Ptilinopus magnificus & Wompoo Fruit-Dove & 17 & 2 & $11.8(2.1-35)$ & M. ptilinopus & under-tail cov. \\
\hline $\begin{array}{l}\text { Ptilinopus } \\
\text { melanospilus }\end{array}$ & Black-naped Fruit-Dove & 3 & 2 & $66.7(13.5-98.3)$ & P. lature & coverts \\
\hline Ptilinopus regina & Rose-crowned Fruit-Dove & 4 & 1 & $25(1.3-75.1)$ & P. lature & coverts \\
\hline Ptilonopus rivoli & White-bibbed Fruit-Dove & 1 & 1 & $100(5-100)$ & M. ptilinopus & under-tail cov. \\
\hline Streptopelia decaocto & Eurasian Collared-Dove & 12 & 2 & $16.7(3-45.7)$ & P. columbae & secondaries \\
\hline Streptopelia capicola & Ring-Necked Dove & NA & NA & - & G. zumpti & contour \\
\hline Streptopelia orientalis & Oriental Turtle-Dove & 22 & 2 & $9.1(1.6-29.1)$ & M. lengai & under-tail cov. \\
\hline $\begin{array}{l}\text { Streptopelia } \\
\text { semitorguata }\end{array}$ & Red-eyed Dove & & 1 & $4.8(0.2-23.3)$ & M. lengai & rectrices \\
\hline $\begin{array}{l}\text { semitorquata } \\
\text { "* }\end{array}$ & & 21 & 1 & $4.8(0.2-23.3)$ & G. zumpti & contour \\
\hline “ & & & 3 & $14.3(4-35.4)$ & P. columbae & coverts \\
\hline Streptopelia turtur & European Turtle-Dove & 30 & 4 & $13.3(4.7-29.8)$ & P. columbae & $\begin{array}{c}\text { contour } \\
\text { under-tail cov. }\end{array}$ \\
\hline Treron waalia & Bruce's Green-Pigeon & 1 & 1 & $100(5.0-100)$ & M. columbicus & covert \\
\hline Turacoena manadensis & White-faced Cuckoo-Dove & 4 & 1 & $25(1.3-75.1)$ & M. turacoenas & under tail cov \\
\hline Turacoena modesta & Black Cuckoo-Dove & 5 & 1 & $20(1-65.7)$ & M. turacoenas & under tail cov \\
\hline Turtur chalcospilos & Emerald-spotted Wood-Dove & 13 & 1 & $7(0.4-34.2)$ & $\begin{array}{c}M . \\
\text { tympanistria }\end{array}$ & coverts \\
\hline Turtur tympanistria & Tambourine Dove & 12 & 2 & $16.7(3-45.7)$ & $\begin{array}{l}M . \\
\text { tympanistria }\end{array}$ & rectrices \\
\hline Zenaida asiatica & White-winged Dove & NA & NA & - & M. zenadourae & - \\
\hline " & & NA & NA & - & T. longisoma & - \\
\hline Zenaida auriculata & Eared Dove & NA & NA & - & M. zenadourae & - \\
\hline Zenaida macroura & Mourning Dove & 1 & 1 & $100(5-100)$ & M. zenadourae & coverts \\
\hline & & NA & NA & - & T. longisoma & primaries \\
\hline
\end{tabular}

Exa.-number of individual host species examined during study; Inf.-number of individual host species, infected by quill mites; $\mathrm{IP}$ - prevalence index given in (\%); CI—confidence interval (Sterne method); NA—infected hosts species, but prevalence index was unknown. *-type host; “- previous species name. 


\subsection{Host Specificity of the Quill Mites}

Based on previously recorded host species, we classified all syringophilids associated with columbiform birds into the following host specificity groups (Tables 3 and 4):

(1) Monoxenous parasites, including 8 species: Gunabopicobia claravis, G. leptotila, G. metriopelia, Meitingsunes adewlles, M. chalcophas, Peristerophila leucomela, Psittaciphilus montanus, Terratosyringophilus geotrygonus.

(2) Oligoxenous parasites, including 5 species: Gunabopicobia geotrygoni, Meitingsunes ptilinopus, M. tympanistria, Psittaciphilus patagioenas, Terratosyringophilus longisoma.

(3) Mesostenoxenous parasites, including 8 species: Gunabopicobia lathami, G. masalaje, G. zumpti, Meitingsunes lengai, M. zenadourae, Peristerophila columbae, P. geopelis, P. lature.

(4) Metastenoxenous parasites, including 3 species: Meitingsunes turacoenas, Peristerophila claravis, Meitingsunes columbicus.

(5) Polyxenous parasites, including only one species: Peristerophila mucuya.

Table 3. Host specificity of quill mite species of the subfamily Syringophilinae with the value of $\mathrm{d}^{\prime}$ index.

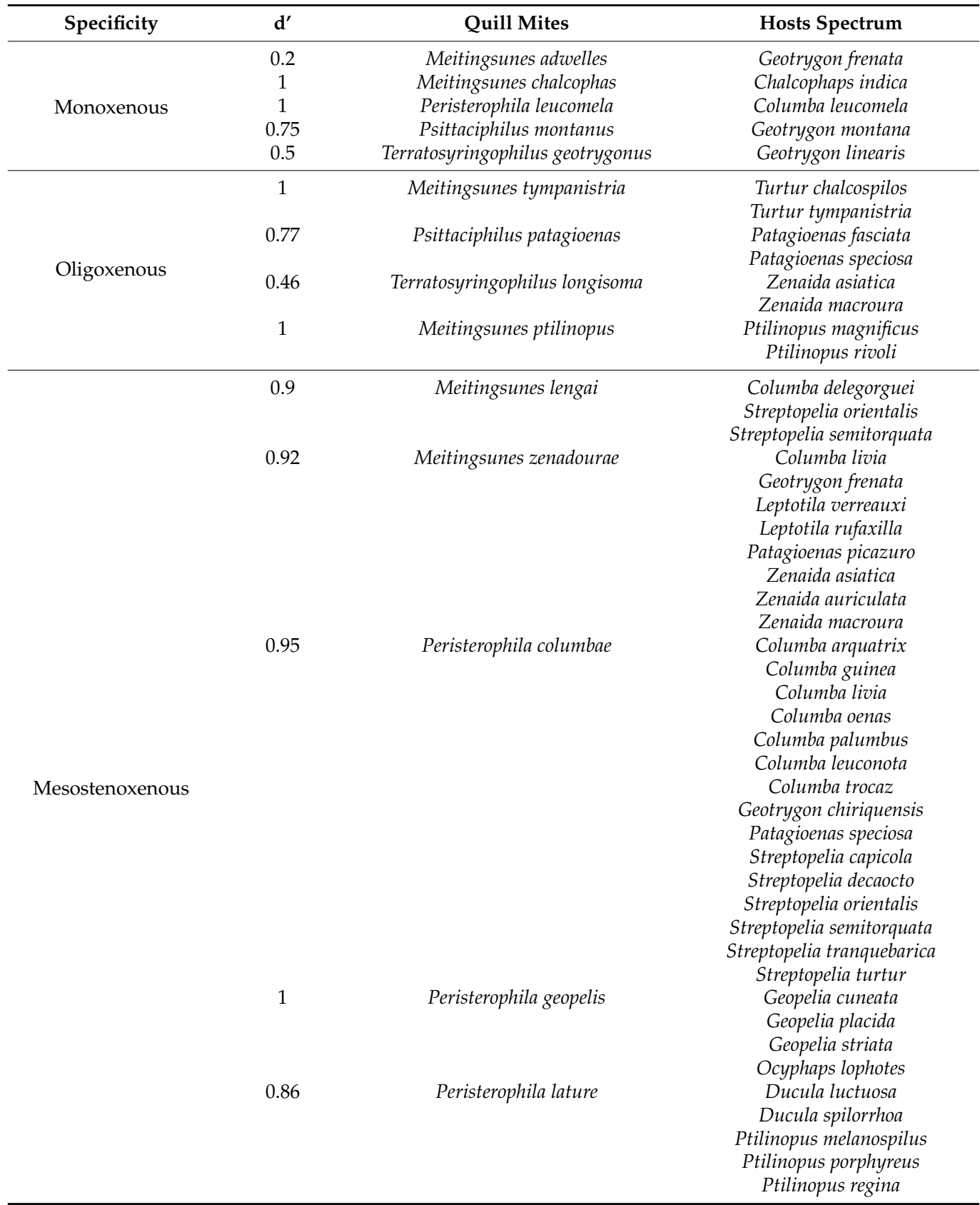


Table 3. Cont.

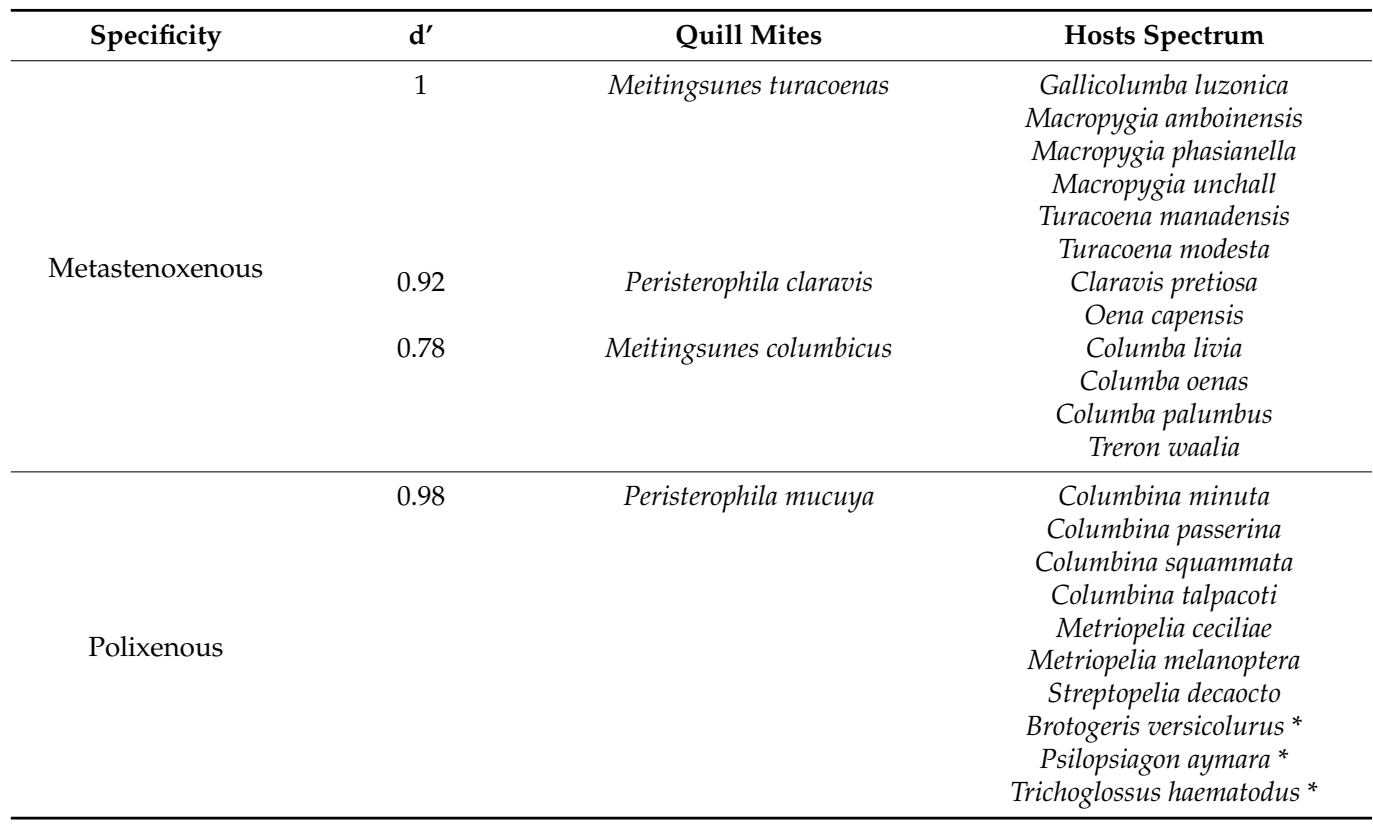

$\mathrm{d}^{\prime}$-index measured specialization at species level; ${ }^{*}$ - hosts species belonging to order Psittaciformes.

Table 4. Host specificity of quill mite species the subfamily Picobiinae with the value of $d^{\prime}$ index.

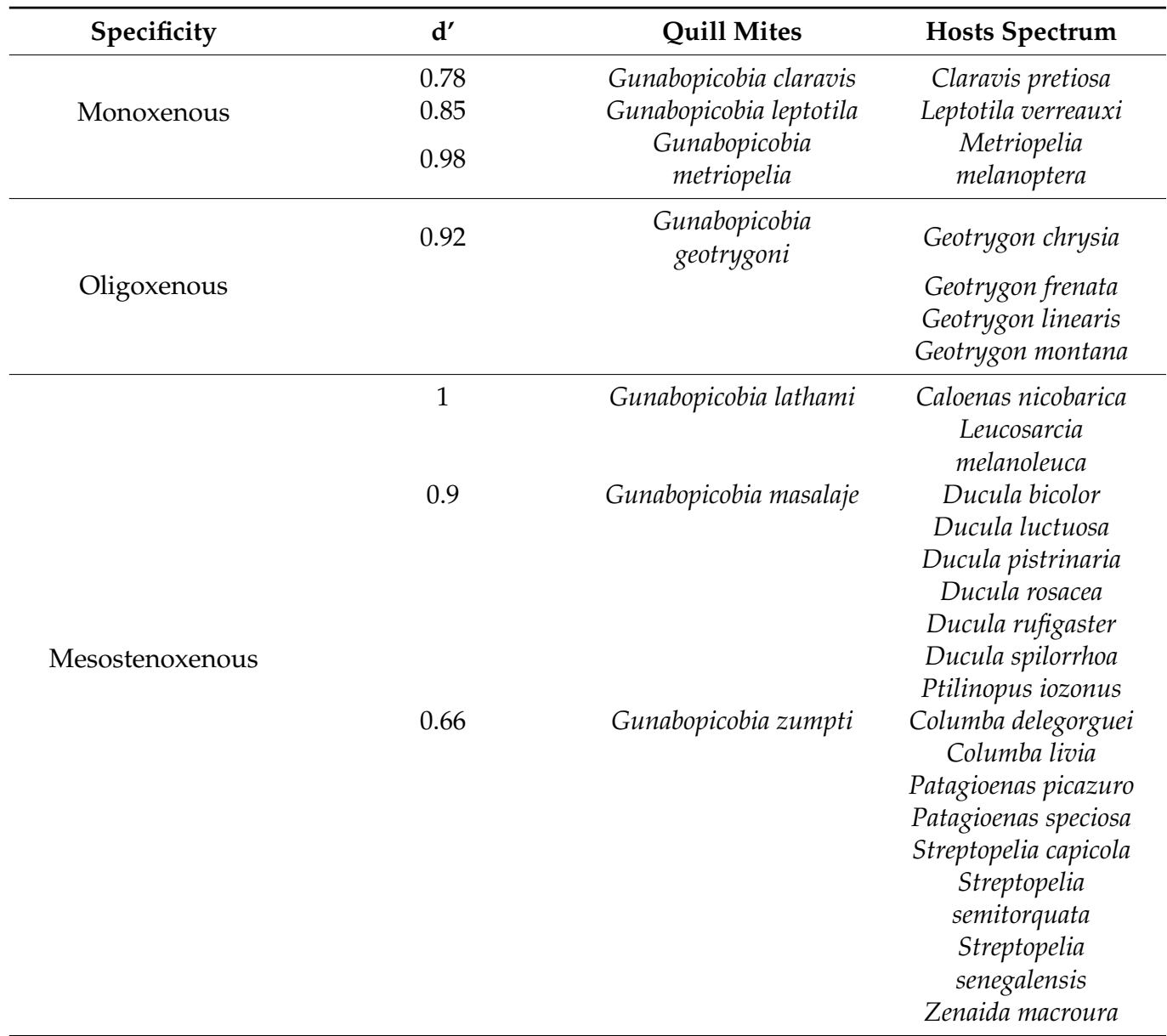

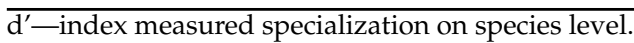




\subsection{Co-Infestation of the Quill Mites}

The analysis of the host spectrum showed several various patterns of co-infestation with niche factor (quill mites occupying a different habitats) (Table 5):

(1) "Syr-Pic" (quill mite species belonging to the differential subfamily Syringophilinae or Picobiinae and inhabiting the same host species but different habitats.

(i) Inhabiting niches: contour feathers (representatives of Picobiinae) and covert (representatives of Syringophilidae): Guanabopicobia claravis + Peristerophila claravis from Claravis pretiosa; G. masalaje + P. lature from Ducula luctuosa and D. spilorrhoa; G. metriopelia + P. mucuya from Metriopelia melanoptera, G. zumpti + P. columbae from Streptopelia semitorquata and Patagioenas speciosa.

(ii) Inhabiting niche: contour feathers (Picobiinae) and under wing coverts (Syringophilidae): G. geotrygoni + M. zenadourae from Geotrygon frenata, G. zumpti + M. zenadourae from Patagioenas picazuro.

(iii) Inhabiting niches: contour feathers (Picobiinae) and under tail coverts (Syringophilidae): G. geotrygoni + Psittaciphilus montanus from Geotrygon, montana, G. leptotila + M. zenadourae from Leptotila verreauxi.

(iv) Inhabiting niches: contour feathers (Picobiinae) and rectrictres (Syringophilidae): G. zumpti + M. lengai.

(2) "Syr-Syr" (different quill mites species belonging to the same subfamily-Syringophilinae and occupying the same host species.

(i) Inhabiting niches: secondaries and covert: Meitingsunes columbicus + Peristerophila columbae from Columba palumbus.

Table 5. Host species infested by two or more syringophilid species with notation of the habitat preference; P-Picobiinae; S-Syringophilinae.

\begin{tabular}{|c|c|c|c|}
\hline Hosts & Quill Mites & Subfamily & Niche \\
\hline \multirow{2}{*}{ Claravis pretiosa } & Gunabopicobia claravis & $\mathrm{P}$ & contour \\
\hline & Peristerophila claravis & $S$ & covert \\
\hline \multirow{2}{*}{ Columba palumbus } & Meitingsunes & $S$ & secondaries \\
\hline & Peristerophila columbae & $S$ & covert \\
\hline \multirow{2}{*}{ Ducula spilorrhoa } & Gunabopicobia masalaje & $\mathrm{P}$ & contour \\
\hline & Peristerophila lature & $S$ & covert \\
\hline \multirow{2}{*}{ Ducula luctuosa } & Gunabopiconia masalaje & $\mathrm{P}$ & contour \\
\hline & Peristerophila lature & S & covert \\
\hline \multirow{2}{*}{ Geotrygon frenata } & Gunabopicobia & $\mathrm{P}$ & contour \\
\hline & Meitingsnes zenadourae & S & under-wing covert \\
\hline \multirow[t]{2}{*}{ Geotrygon montana } & $\begin{array}{l}\text { Gunabopicobia } \\
\text { geotrygoni }\end{array}$ & $\mathrm{P}$ & contour \\
\hline & $\begin{array}{l}\text { Psittaciphilus } \\
\text { montanus }\end{array}$ & $S$ & under-tail covert \\
\hline \multirow{2}{*}{ Leptotila verreauxi } & Gunabopicobia leptotila & $\mathrm{P}$ & contour \\
\hline & $\begin{array}{l}\text { Meitingsunes } \\
\text { zenadoure }\end{array}$ & $S$ & under tail-covert \\
\hline \multirow{2}{*}{$\begin{array}{l}\text { Metriopelia } \\
\text { melanoptera }\end{array}$} & $\begin{array}{l}\text { Gunapopicobia } \\
\text { metriopelia }\end{array}$ & $\mathrm{P}$ & contour \\
\hline & Peristerophila muсuуa & $S$ & covert \\
\hline
\end{tabular}


Table 5. Cont.

\begin{tabular}{cccc}
\hline Hosts & Quill Mites & Subfamily & Niche \\
\hline Patagioenas picazuro & $\begin{array}{c}\text { Gunabopicobiazumpti } \\
\text { Meitingsunes } \\
\text { zenadourae }\end{array}$ & $\mathrm{P}$ & contour \\
& Gunabopicobia zumpti & $\mathrm{S}$ & under-wing covert \\
Patagioenas speciosa & Peristerophila columbae & $\mathrm{S}$ & contour \\
& Psittaciphilus & $\mathrm{S}$ & covert \\
& patagioenas & & covert \\
\hline Streptopelia & Gunabopicobia zumpti & $\mathrm{P}$ & contour \\
semitorquata & Meitingsunes lengai & $\mathrm{S}$ & rectrices \\
& Peristerophila columbae & $\mathrm{S}$ & covert
\end{tabular}

Subfamily of the family Syringophilidae: (P)_Picobiinae, (S)-Syringophilidae.

Moreover, in one sample, we observed quill mite species belonging to the same subfamily and inhabiting the same host species, and moreover occupying the same type of feathers. This pattern was found in Peristerophila columbae + Psittaciphilus patagioenas where both species occupied covert feathers of Patagioenas speciosa (Table 4).

\subsection{Bipartite Network Analysis}

The Columbiformes-Syringophilidae bipartite network (Figure 1) had high connectance $(\mathrm{C}=0.90)$ and high specialization $\left(\mathrm{H}^{\prime}{ }^{\prime}=0.93\right)$ with a high degree of nestedness (0.908). The comparison between $\mathrm{H} 2^{\prime}$ and null model values, showed significant differences (mean for null model $=0.56 ; p=0.0009271$ ).

We also measured specialization on the species-level ( $\left.d^{\prime}\right)$. Quill mites specialization ranged between 0.20 and 1 (see Tables 3 and 4).

(1) d' 0.1-0.59: M. adwelles (0.2), T. longisoma (0.46), T. geotrygonus (0.5).

(2) d' 0.6-0.99: G. zumpti (0.66), P. montanus (0.75), P. patagioenas (0.77), M. columbicus (0.78), G. claravis (0.78), G. leptotila (0.85), P. lature (0.86), M. lengai (0.9), G. masalaje (0.9), M. zenadourae (0.92), G. geotrygoni (0.92), P. claravis (0.92), P. columbae (0.95), G. metriopelia (0.98), P. muсuya (0.98).

(3) d' = 1: G. lathami, M. chalophaps, M. tympanistria, M. turacoenas, M. ptilinopus, P. geopelis, P. leucomela.

The strength (thickness of connecting bar between parasites and hosts) of each interaction is representative of the number of interactions (prevalence). Each link corresponds to species interaction and represent quill mites genera: red-Gunabopicobia, blue-Meitingsunes, black-Terratosyringophilus, green-Psittaciphilus, yellow-Peristerophila. Host phylogeny based on Jetz et al. [68].

We registered a high modularity (likelihood $=0.83$ ) with 20 modules. Modules were split to (A) single-host (quill mites associated with one host species), (B) multi-host (quill mites associated with more the one host species), and (C) multi-parasites (modules encompasses more than one quill mite species) modules (Figure 2).

A. Single-host module: (1) Gunabopicobia leptotila—Leptotila verrauxi, (2) Gunabopicobia metriopelia-Metriopelia melanoptera, (6) Peristerophila leucomela-Columba leucomela, (14) Terratosyringophilus longisoma-Zenaida asiatica, (16) Meitingsunes chalcophas-Chalcophaps indica.

B. Multi-host module: (4) Gunabopicobia masalaje-(Ducula bicolor, Ducula rufigaster, Ducula rosacea, Ducula pistrinaria, Ptilinopus iozonus); (5) Gunabopicobia lathami-(Leucosarcia melanoleuca, Caloenas nicobarica); (7) Meitingunes columbicus-(Columba palumbus, Treron waalia); (8) Meitingsunes tympanistria-(Turtur chalcospilos, Turtur tympanistria); (10) Meitingsunes zenadourae-(Leptotila rufaxilla, Patagioenas picazuro, Zenaida auriculata, Zenaida macroura); (11) Meitingsunes turacoenas (Gallicolumba luzonica, Macropygia amboinensis, Macropygia phasianella, Macropygia unchall, Turacoena manadensis, Turacoena modesta); (12) Meitingsunes psittaciphilus 
(Ptilinopus magnificus, Ptilinopus rivoli); (13) Psittaciphilus patagioenas-(Patagioenas fasciata, Patagioenas speciosa); (15) Peristerophila geopelis (Geopelia cuneata, Geopelia placida, Geopelia striata, Ocyphaps lophotes); (17) Peristerophila lature (Ducula luctuosa, Ducula spilorrhoa, Ptilinopus jambu, Ptilinopus melanospilus, Ptilinopus porphyreus, Ptilinopus regina); (19) Peristerophila columbicus (Columba arguatrix, Columba guinea, Columba livia, Columba oenas, Columba trocaz, Columba leuconota, Geotrygon chiriquensis, Streptopelia decaocto, Streptopelia semitorquata, Streptopelia turtur); (20) Peristerophila mucuya (Columbina minuta, Columbina passerina, Columbina squammata, Columbina talpacoti, Metriopelia ceciliae).

C. Multi-parasite module: (18) Peristerophila claravis-Gunabopicobia claravis Gunabopicobia geotrygoni-Meitingsunes zenadourae-Psittaciphilus montanus-Meitingsunes columbicus; (9) Gunabopicobia zumpti-Meitingsunes lenagi.

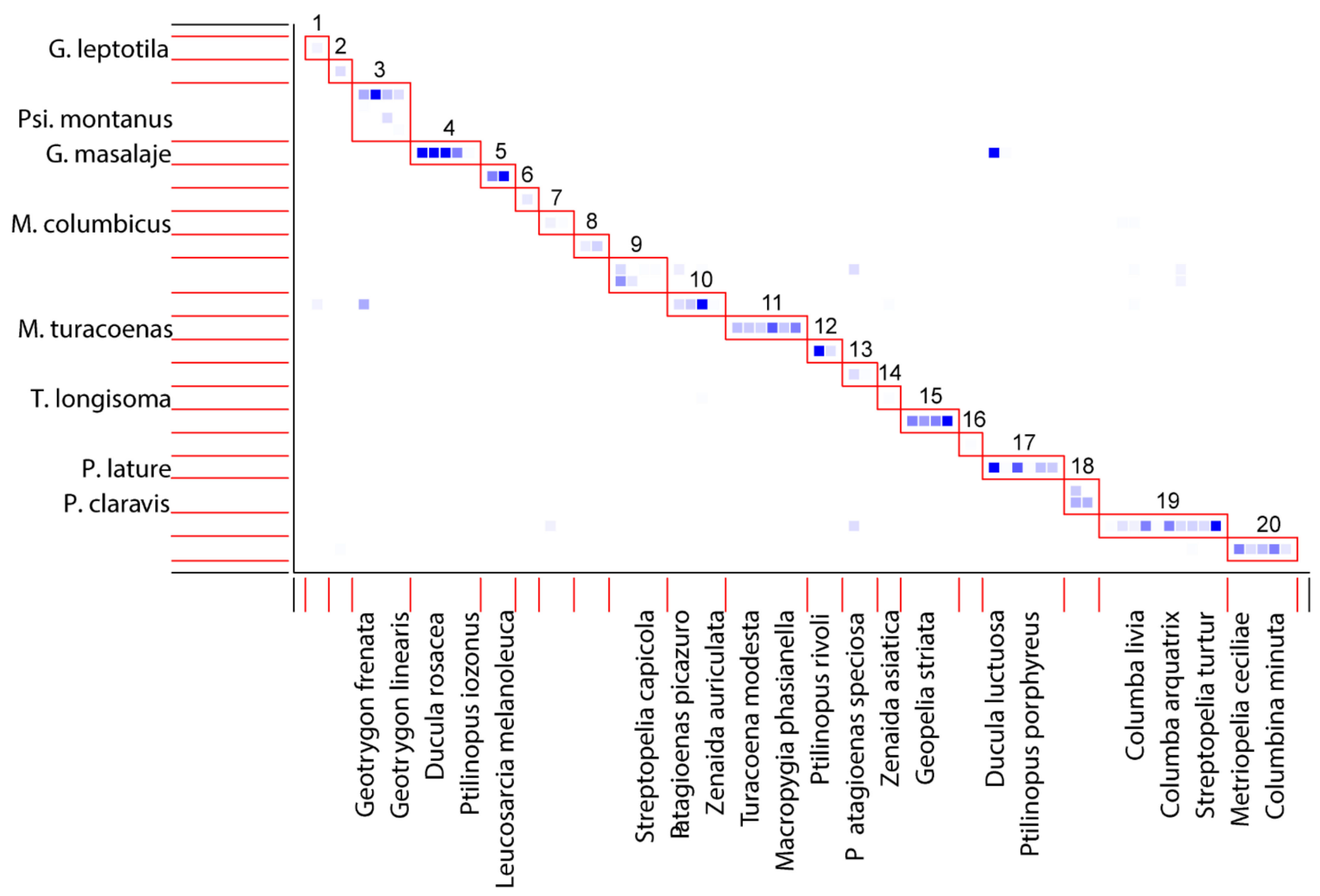

Figure 2. Modules of the quill mites-doves communities. Modules 1-20, generated for quill mites species and doves and pigeons. The intensity of the colors of the squares indicates the strength of the interaction, between particularly parasites species (vertical axis) and their hosts species (horizontal axis).

\subsection{Zoogeographical Distribution of Quill Mite Species Associated with Pigeons and Doves}

Based on previous reports (see Table 1), we summarized the distribution of the Syringophilidae associated with birds from order Columbiformes. Quill mite species were recorded in hosts inhabiting the following zoogeographical regions: Neotropical, Nearctic, Panamanian, Palaearctic, Saharo-Arabian, Afrotropical, Oriental, Australasian, and Oceanian (Table 6, Figure 3). In particular regions, we noted the following genera with number of quill mites species: 
- Neotropical: Gunabopicobia (5), Meitingsunes (2), Peristerophila (3), Psittaciphilus (2), Terratosyringophilus (1);

- $\quad$ Nearctic: Meitingsunes (1), Peristerophila (2), Terratosyringophilus (1), Gunabopicobia (1);

- Panamanian: Psittaciphilus (1), Peristerophila (2), Gunabopicobia (1);

- Palaearctic: Meitingsunes (8), Peristerophila (2), Gunabopicobia (1);

- Saharo-Arabian: Peristerophila (1);

- Afrotropical: Meitingsunes (4), Peristerophila (2), Gunabopicobia (1);

- $\quad$ Oriental: Meitingsunes (2), Peristerophila (4), Gunabopicobia (2);

- Oceanian: Meitingsunes (2), Peristerophila (1), Gunabopicobia (2);

- Australasian: Meitingsunes (2), Peristerophila (4).

Table 6. Distribution of syringophilid associated with birds from order Columbiformes in zoogeographical regions.

\begin{tabular}{|c|c|c|c|c|c|c|c|c|c|}
\hline \multicolumn{10}{|c|}{ Zoogeographic Regions } \\
\hline $\begin{array}{l}\text { Quill Mites } \\
\text { Species }\end{array}$ & Neot. & Near. & Pana. & Pala. & Sa-Ara. & Afro. & Orie. & Ocean. & Austr. \\
\hline \multicolumn{10}{|l|}{ M. aldwelles } \\
\hline \multicolumn{10}{|l|}{ M. columbicus } \\
\hline \multicolumn{10}{|l|}{ M. chalcophas } \\
\hline \multicolumn{10}{|l|}{ M. ptilinopus } \\
\hline \multicolumn{10}{|l|}{ M. lengai } \\
\hline \multicolumn{10}{|l|}{ M. turacoenas } \\
\hline \multicolumn{10}{|l|}{ M. tympanistria } \\
\hline \multicolumn{10}{|l|}{ M. zenadourae } \\
\hline \multicolumn{10}{|l|}{ P. columbae } \\
\hline \multicolumn{10}{|l|}{ P. claravis } \\
\hline \multicolumn{10}{|l|}{ P. geopelis } \\
\hline \multicolumn{10}{|l|}{ P. lature } \\
\hline \multicolumn{10}{|l|}{ P. leucomela } \\
\hline \multicolumn{10}{|l|}{ P. muсuуa } \\
\hline \multicolumn{10}{|l|}{ P. montanus } \\
\hline \multicolumn{10}{|l|}{ P. patagioenas } \\
\hline \multicolumn{10}{|l|}{ T. geotrygonus } \\
\hline \multicolumn{10}{|l|}{ T. longisoma } \\
\hline \multicolumn{10}{|l|}{ G. claravis } \\
\hline \multicolumn{10}{|l|}{ G. geotrygoni } \\
\hline \multicolumn{10}{|l|}{ G. masalaje } \\
\hline \multicolumn{10}{|l|}{ G. metriopelia } \\
\hline \multicolumn{10}{|l|}{ G. lathami } \\
\hline \multicolumn{10}{|l|}{ G. leptotila } \\
\hline G. zumpti & & & & & & & & & \\
\hline
\end{tabular}

Zoogeographical regions: Afro.-Afrotropical, Aust.—Australian, Near.-Nearctic, Neot.-Neotropical, Ocea.-Oceanian, Orie.-Oriental, Pala.-Palaearctic, Pana._Panamanian, Sa-Arab.-Saharo-Arabian, Si-Jap.-Sino-Japanese (according to Holt et al. [71]).

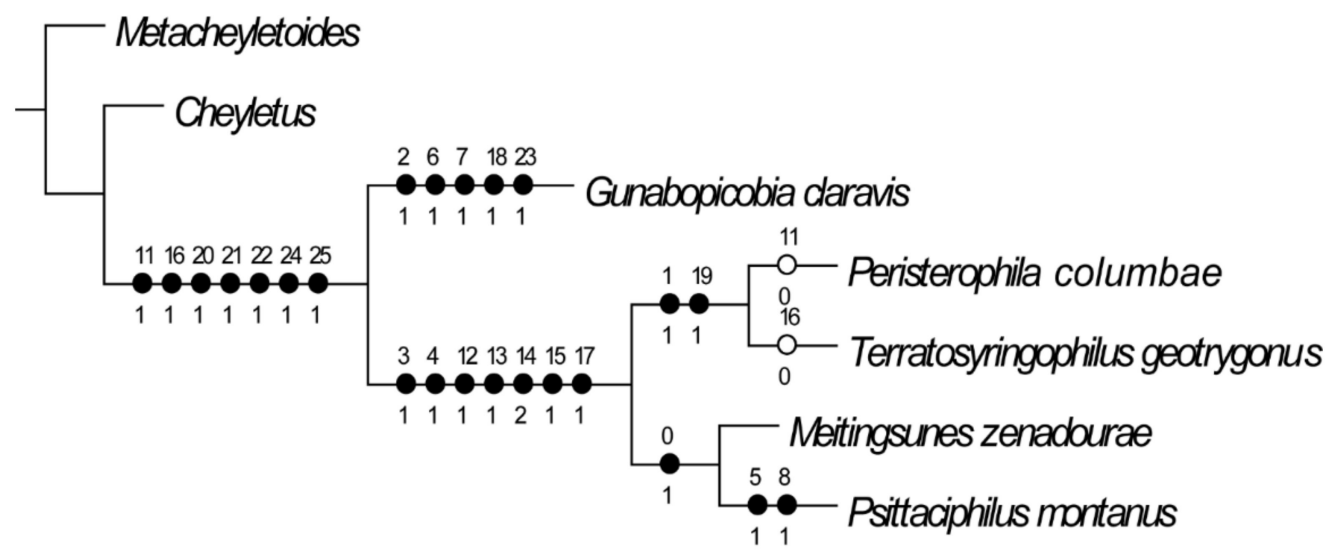

Figure 3. Phylogenetic tree of selected genera of Syringophilidae mites associated with Columbiformes birds. 
Among all quill mites species, eight of them were only noted from one region: Neotropical-Gunabopicobia metriopelia, G. claravis, G. leptotila, Meitingsunes adwelles, Psittaciphilus patagioenas Terratosyringophilus geotrygonus; Afrotropical-Meitingsunes tympanistria; Australian-Peristerophila leucomela. Others quill mites species were recorded from more than one zoogeographical region:

- Neotropical + Nearctic + Palaearctic + Afrotropical: Gunabopicobia zumpti; Meitingsunes zenadourae; Peristerophila claravis;

- Neotropical + Nearctic: Psittaciphilus patagioenas;

- Palaearctic + Afrotropical: Meitingsunes columbicus, Meitingsunes lengai;

- Oriental + Oceanian: Meitingsunes turacones;

- Oriental + Australasian: Meitingsunes chalcophas;

- Oceanian + Oriental + Australasian: Peristerophila lature;

- Neotropical + Nearctic + Palaearctic + Afrotropical + Saharo-Arabian + Oriental: Peristerophila columbae.

\subsection{Phylogenetic Analysis}

The analysis under equal weights resulted in one most parsimonious tree (MPT) shown in Figure 4. Number of characters-26 (Figures S2 and S3), number of parsimonyinformative characters-19, tree length $(\mathrm{L})=30$, consistency index $(\mathrm{CI})=0.9$, retention index $(\mathrm{RI})=0.9$, rescaled consistency index $(\mathrm{RC})=0.8$, homoplasy index $(\mathrm{HI})-0.1$, Goloboff-fits $(\mathrm{G}$-fit $)=-18.25$.
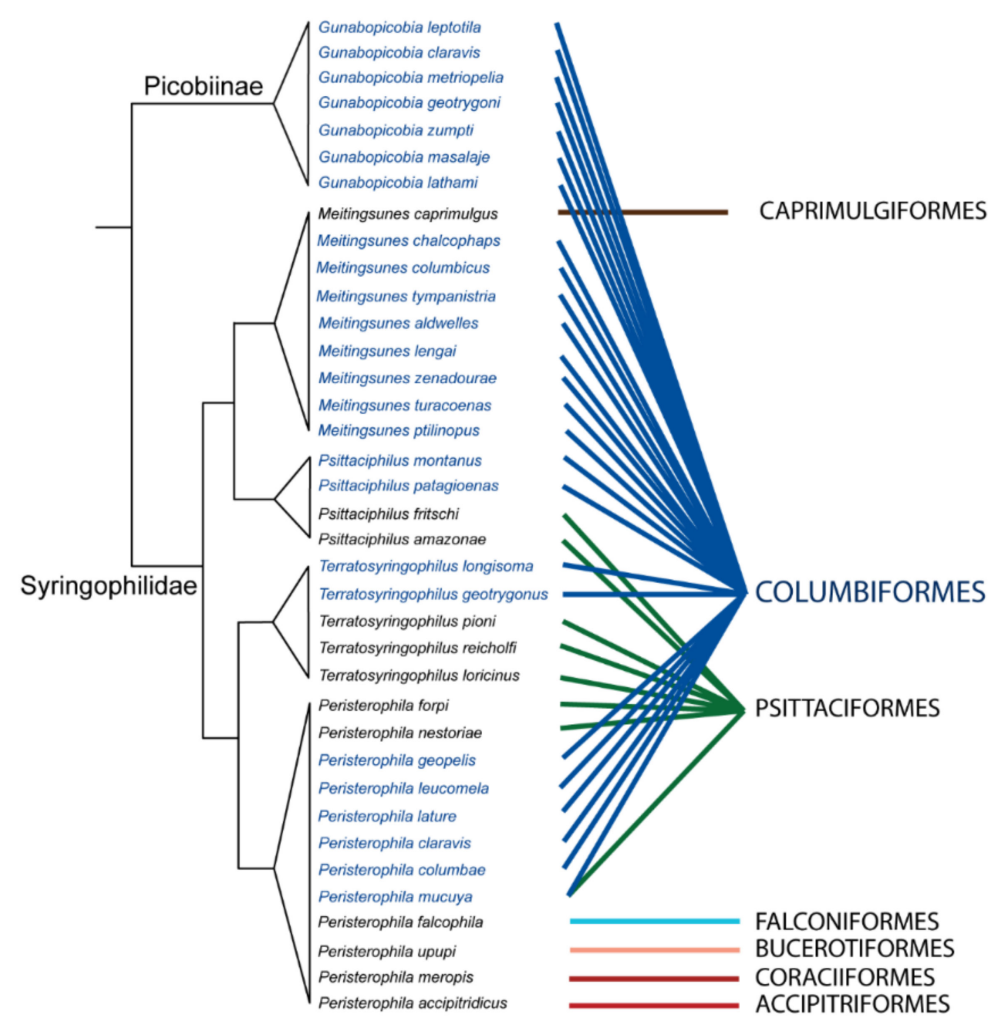

Figure 4. Phylogenetic tree of selected genera of Syringophilidae associated with differential hosts order.

The analysis shows that except the genus Gunabopicobia which represents subfamily Picobiinae, other syringophilinae genera form two distinct clades: Peristerophila + Terratosyringophilus (supported by synapomorphies: the presence of large finger-like protuberances on the hypostomal apex, presence of parallel apodemes I, and presence of dimorphic females) and Meitingsunes + Psittaciphilus (supported by synapomorphy: the presence of the constricted posterior end of the stylophore. 


\section{Discussion}

The parasitological studies on quill mites of the family Syringophilidae and their hosts have a long history spanning over 140 years [16,22]. However, the extensive studies on this group of parasites started about 40 years ago, and investigation of a small fraction of the about 10,000 extant bird species recognized to date recording of more than 400 species of syringophilid mites arranged in 63 genera and two families [20].

The studies on the host-parasite relationship in the system composed of quill mites and the particular taxonomical groups of their hosts are still rare in the literature. Moreover, comprehensive research of the quill mite fauna on the host representatives of the whole bird order and considering mite species richness, host and habitat specificities, prevalence, and phylogenetic relationships have not been provided so far. Most of the previously published papers have focused on describing syringophilid fauna of the particular zoogeographical regions e.g., [22,23,77], or on taxonomical reviewing the different taxa (genus or subfamily) of quill mites e.g., $[23,26,41,78]$. Recently, however, there have been published a few studies examining the syringophilid fauna on the particular taxonomical host groups (e.g., passeriform genus Estrilda [25], sub-Saharan Nectariniidae [24], cuckoos [79]), with primary analyses of host-parasite relationships.

This paper focuses on analyses of the species richness and measuring specialization and interaction between syringophilid mites parasitizing columbiform birds in their natural host-parasite system.

\subsection{Species Richness and Phylogenetic Relationship of Quill Mites Associated with Columbiform Birds}

The fauna of quill mites associated with doves and pigeons encompasses 25 species belonging to the following five genera: Meitingsunes, Peristerophila, Psittaciphilus, Terratosyringophilus (subfamily Syringophilinae), and Gunabopicobia (subfamily Picobiinae) (see Table 1). Among them, only one-Gunabopicobia-is exclusively associated with columbiform birds and represented by monoxenous ( 3 species), oligoxenous (1), and mesostenoxenous (3) parasites. Thus, this genus is a perfect example of the host-parasite interaction where a supraspecific taxon of parasites is associated with one host order. This genus is known from hosts representing all columbiform subfamilies, i.e., Claravinae, Columbinae, and Raphinae. It was suggested by Kaszewska et al. [26] that mites of this genus could have started to parasitize the common ancestor in the Late Cretaceous (about 41 to 46 MYA) before their split on the particular subfamilies. Moreover, the Columbiformes are one of the oldest lineages of extant birds. A recent molecular study based on the complete mitochondrial genome suggests that the earliest radiation of the Columbidae occurred during the late Oligocene and continued diversification of the major clade in the Miocene [15]. However, older data suggest that the Columbiformes radiated from Eocene to Oligocene [13,14] or even from Early Eocene to middle Miocene [12].

Four genera of the syringophilines associated with columbiform birds have been previously assigned to the Psittaciphilus-generic-group [80]. In this study, we identified phylogenetically closely related clades Meitingsunes + Psittaciphilus and Peristerophila + Terratosyringophilus.

The genus Meitingsunes comprises nine described species where eight of them are exclusively associated with pigeons and doves infesting birds belonging to two subfamilies Columbinae and Raphinae (28 infested species in total) [45]. However, only one species of this genus, M. caprimulgus, has been noted from the phylogenetically distant clade of nightjars (Caprimulgiformes) [50]. Because birds belonging to the order Caprimulgiformes are extremely poorly examined (with only one host record), the status of Meitingsunes on nightjars is still unclear. The nightjars can represent real hosts for quill mites of this genus, or the single findings of $M$. caprimulgus can be an example of host-switching (e.g., from the columbiform host).

The genus Psittaciphilus includes four species found on representatives of Columbiformes (2 species) and Psittaciformes (2) [33,42]. On pigeons and doves, this genus infests 
birds of the genera Geotrygon and Patagioenas, which are also parasitized by members of the genus Meitingsunes mentioned above (e.g., M. zenadourae and M. adwelles).

The genus Terratosyringophilus includes three quill mites species found on parrots and two species noted from doves belonging to the subfamily Columbinae $[31,34,35,37,81]$.

The Terratosyringophilus quill mites along with Psittaciphilus and partially Peristerophila (see below) have been found in birds from orders Columbiformes and Psittaciformes. The cases where both host orders are infested by mites belonging to the same genera can indicate the phylogenetically close relationship between these two bird orders. However, recent phylogenetic analysis does not confirm this hypothesis. It is commonly accepted that the lineage of doves and pigeons is a sister clade to sandgrouse (Pteroclidiformes) and mesites (Mesitornithiformes) [15,82-84]. At this moment, we cannot explain this multi-order infestation of the same genera of syringophilid mites. To resolve this problem, the molecular analyses of the quill mites phylogeny are needed as well as the studies on the host spectrum of the other symbionts parasitizing birds of these both orders.

The genus Peristerophila comprises 14 quill mites species and is the only genus that inhabits not only doves and parrots but also hosts belonging to hawks (Accipitriformes), falcons (Falconiformes), hoopoes (Bucerotiformes), rollers and bee-eaters (Coraciiformes) $[43,46,48,75,85,86]$. The Peristerophila mites associated with columbiform birds are recorded on hosts from all subfamilies, i.e., Columbinae, Raphinae, and Claravinae. Moreover, among all 30 species of syringophilines recorded on pigeons and doves, there is only one species-Peristerophila mucuya, representing polyxenous parasite-which is found on hosts belonging to the order Columbiformes and Psittaciformes (Figure 4).

\subsection{Columbiform Hosts and Quill Mite Fauna}

Our study of the quill mites associated with columbid birds was conducted on doves and pigeons representing all subfamilies, i.e., Columbinae, Claraviinae, and Raphinae. Based on material used for our research and records from previous publications, we estimated that the degree of species testing of Columbiformes ranged from $25 \%$ to $100 \%$ (for individual genera). In the subfamily Columbinae, regarding investigation degree of host species, more than $50 \%$ is in the following host genera: Streptopelia (52\%), Turacoena (66\%), Zenaida $(85 \%)$, Leptotrygon (100\%). In the subfamily Claravinae, we examined all currently recognized genera except monotypic genus Uropelia, Claravis (100\%), Columbina (55\%), Metriopelia (50\%), Paraclaravis (50\%). In the subfamily Raphinae, investigation degree more than $50 \%$ is in the following host genera: Caloenas (50\%), Chalcophas (66\%), Geopelia (60\%), Henicophaps (100\%), Leptotrygon (100\%), Leucosarcia $(100 \%)$, Ocyphaps $(100 \%)$, Oena $(100 \%)$, Otidiphaps (100\%).

Considering high-level examination of columbiform birds under the presence of the quill mites, we suppose that the Syringophilidae fauna on the generic level has been fully explored. In the future, it would be worth intensifying research on the syringophilids inhabiting a single bird order. It will allow comparing our results with these ones conducted for other host orders. This approach allows for a better understanding of the parasite-host relationship as a whole. It would also be interesting to provide comprehensive studies on quill mite fauna associated with pacific island doves and pigeons. The future collection of the material from these regions will allow testing MacArthur and Wilson's "the island theory" for quill mites. Additionally, future molecular studies on co-phylogeny also give important information about the relationships and evolutionary events between particular columbid and quill mite species.

\subsection{Prevalence}

The prevalence index provided details of the strength of the relationship between a particular host and parasites species. Our study has shown that the prevalence of infested birds by the quill mites ranges between $4.2 \%$ and $66.7 \%$. However, for 17 hosts species, IP was equal to $100 \%$, the confidence interval (CI) was wide, and this result can be the effect of the small sample size of studied host specimens. 
The highest prevalences were detected in the previous studies for birds kept on the farms, e.g., domestic hen Gallus gallus domesticus infested by Syringophilu bipectinatus Heller, where IP was 75\% ( $\mathrm{N}=1.500)$ [87] or for social species, e.g., house sparrow Passer domesticus infested by Syringophiloidus minor where IP was $82 \%(\mathrm{~N}=492)$ [88]. For non-social birds, the prevalence index is much lower. It usually does not reach $50 \%$ (see works on prevalence among various passerine species (IP varies between $3.5 \%$ and $42.9 \%$ ) $[24,25,89-93]$; phasianids (IP = 5.5-7.3\%) [94-96]; parrots (IP = 7.7-20\%) [97].

Both factors, the number of examined bird individuals and the number of examined feathers, play a crucial role in determining the real prevalence of infested hosts in the environment. In current and previous studies on prevalence, the used bird material was from various sources. The first source includes birds deposited in the museum collections (mostly dry bird skins and frozen or alcohol preserved specimens) e.g., [24,25]. The second source are birds examined during fieldworks (e.g, [18,48,88,90,92,93,95,98-101]) or kept in the zoological gardens [97] and farms [94,96,102].

It is obvious that syringophilid mites infest not all host specimens in nature and not all feathers, and to present the real IP, we should examine as many as possible bird individuals (taking into consideration their age, season, locality, etc.) and as many as possible feathers; see also $[93,95,103]$. However, samples collected from ornithological collections and from live birds are limited and allow sampling only a few feathers. Therefore, to minimize this limiting factor, we should continue studies on habitat specificity (see below).

\subsection{Habitat Specificity and Multi-Infestation of Syringophilid Mites}

The feather environment gives opportunities to inhabit various niches by ectoparasites and commensal species. However, the phenomenon of co-infestation remains poorly documented, especially for ectoparasites belonging to the family Syringophilidae. The first remark about multi-infestation was pointed out by Kethley [16]. He indicated that one host species or even one host individual may be infected by several syringophilid species inhabiting different types of feathers. Later on, Schmäschke et al. [104] presented the observation of co-infestation of two species, Syringophilopsis turdi and Syringophiloidus sp. on one the fieldfare Turdus pilaris (Passeriformes: Turdidae), and Syringophilopsis kirgizorum and Syringophiloidus sp. found on the greenfinch Carduelis chloris (Passeriformes: Fringillidae). Other examples of multi-infestations were described by Skoracki et al. [91]. In this paper, the authors recorded the following patterns of infestation with the notation of infested niches, e.g., Torotrogla rubeculi (habitat: secondaries) + Picobia sp. (habitat: contour feathers) on the European robin Erithacus rubecula (Muscicapidae); Syringophilipsis kirgizorum (primaries) + Torotrogla gaudi (secondaries) on the chaffinch Fringilla coelebs (Fringillidae); Syringophiloidus presentlis (secondaries) + Picobia sturni and Aulonastus buczekae (habitat: contour feathers) on the common starling Sturnus vulgaris (Sturnidae).

Until now, the multi-infestations by quill mites have been observed only in passeriform birds. However, our study described other cases of syringophilid multi-infestation on columbiform birds and showed that the phenomenon of co-infestation can occur more frequently. In total, we found 13 examples of co-infestation in different configurations. The most frequent cases of co-infestation were recorded for quill mites that inhabited the same host species but occupied differential niche-"factor niche". For these cases, we observed two co-infestation patterns: (1) "Syr-Pic pattern"—quill mites belonging to two subfamilies Syringophilinae and Picobiinae occupying the same host individual or species; and (2) "Syr-Syr pattern"—quill mites belonging to the same subfamily, Syringophilinae. Currently, the pattern "Pic-Pic", i.e., two species of picobiine mites on the same host species, was not observed. In members of the "Syr-Pic pattern", representing two subfamilies, differences in morphology, life strategies, and niche preferences are clearly visible. For example, Picobiinae inhabit exclusively contour feathers while the members of Syringophilinae occur mainly inside the quills of secondaries, wing or tail coverts, rectrices; however, they are also occasionally found in contour feathers (Tables 2 and 5). For the "Syr-Syr pattern", we observed a similar strategy of avoiding competition by occupying different feathers. 
However, for this group, we found two species, Peristerophila columbae and Psittaciphilus patagioenas, that infested the same host species and occupied the same niche-quills of wing coverts. Probably, this event could be an example of the horizontal transfer.

Niche separation among quill mites is a result of avoiding competition for the same microhabitat. According to the niche conception, the differential species cannot occupy the same niche (and use the same resources) because the advantage for one competitor will eventually drive others to extinction [105-107]. Finally, niche separation is the process of natural selection which drives competing species into using different hosts or different microhabitats [108].

Examples of niche separation are common and well documented for other ectoparasitic mites, e.g., mites from genus Schizocarpus infested Castor fiber [109] or feathers mites such as Microspalax brevipes and Zachvatkinia ovata associated with Calonectris borealis [110]. However, knowledge about competition and niche overlap phenomena for ectoparasites of the Syringophilidae is still unsuccessfully documented. The following examples of coinfestation in syringophilid groups provided in our study confirm the previous reports on the high degree of specificity of the quill mites to occupying niche. The observed preferences of syringophilids to colonize various types of feathers can result from the preferences to the specific parameters of the quills, such as the thickness of the quill wall and its volume. This hypothesis was proposed by Kethley [17], Casto [18], and Glowska et al. [111] Moreover, recent studies by Grossi and Proctor [93] confirmed a strong correlation between quill volume and the average number of quill mites.

\subsection{Bipartite Network of the Quill Mites-Doves Communities}

The ecological network approach provides a lot of information about biological systems. Networks can be useful to illustrate and analyze the relationships and ecological interactions inside various types of communities [8]. Recently, an extensive study of an ecological network aimed to describe the character of mutualistic plant-animal interactions (pollination, seed dispersal, etc.) $[5,56,112,113]$. However, the network-thinking approach may also be useful in the study of the parasite ecology. Those analyses give a visual graph that illustrates links between two trophic levels, but above all, quantify indices such as host specificity in parasites and provide the topological description [5,9].

Network analyses were conducted for host-parasite systems, e.g., herbivorous insectsparasitoid food web [114] or tropical bats and their ectoparasitic bloodsucking flies [113]. Until now, bipartite analyses have been used for quill mites associated with the following host groups: sunbirds (Passeriformes: Nectariniidae) [24], estrildids (Estrildidae) [25], and doves (Columbiformes: Columbidae) [26].

In the present study, to describe the bipartite network, we used the following indices: connectance $(\mathrm{C})$, nestedness $(\mathrm{N})$, modularity $(\mathrm{Q})$, and $\mathrm{H} 2$ '. The values of these metrics provided information about: the number of interactions, the level of sharing partners, the degree of compartmentalization of the networks, and network-level specialization $[52,115,116]$. Our results confirm the hypothesis about the high specialization of syringophilid mites associated with pigeons and doves. We found strong specialization on both the network- and the species-level. The architecture of the quill mites-doves network was characterized by a high: connectance $(\mathrm{C}=90)$, nestedness $(\mathrm{N}=0.908), \mathrm{H} 2^{\prime}\left(\mathrm{H} 2^{\prime}=0.93\right)$, and also with simultaneously high value of modularity $(Q=0.83)$ with 20 modules.

Recent studies of ecological networks have shown that the metrics such as nestedness, modularity, and connectance are correlated and depend on one another $[57,58,117]$, which can be useful to understand the interaction between particular species in the network. One of the most important indices used to describe the quill mites-doves network was nestedness. We noted a high value of $(\mathrm{N})=0.908$, close to 1 . According to Bascompte et al. [56], the results close to 1 indicate a non-random community structure with a high level of diversity and complexity. Moreover, quill mites-doves communities were shown to have a highly modular structure. Modularity measures the tendency of a network to divide into modules (also called groups, clusters, or communities) [57]. It promotes stability by 
containing perturbations within a module, thereby constraining their spreading to the rest of the community [118]. In our networks, we found 20 modules, each of them had a strong interaction between species inside the modules. Some recognized modules (Figure 2) have more than one quill mite species, e.g., module number " 3 " had the highest number of quill mites species: G. geotrygoni, M. zenadourae, P. montanus, and M. columbicus. These multi-parasite communities interact with numerous hosts and probably can result from the phylogenetic relationship between particular quill mites and their hosts. The genera Psittaciphilus and Meitingsunes are sister clades (Figure 3) within subfamily Syringophilinae and share the same close relation to host species, while the genus Gunabopicobia is a separately evolutionary line. Moreover, those results suggest the structure of communities where competition for hosts can be expected. We observed another situation for modules where only one quill mite species has infested one host species. Those communities are represented, for example, by Gunabopicobia metriopelia associated with one host species, Metriopelia melanoptera. In this case, strong interaction with hosts species was observed.

The next indicator of complexity-connectance-was used in this study. The strong link between parasitic species and individual hosts $(C=0.90)$ observed in our research may be the result of non-random infestation.

The similar architecture of the bipartite network was presented in a study of ectoparasitic flies of the family Streblidae (Hippoboscoidea) and bat hosts from the tropical dry forest [113]. The authors of this study obtained structures similar to ours, such as high specialization $\left(\mathrm{H} 2^{\prime}=0.67\right)$, high modularity $(\mathrm{Q}=0.7)$, but, contrary to the quill mites-doves nest, the authors found a low value of connectance $(C=0.30)$. The differential between $\mathrm{C}$ index can be related to sampled and network size. This relation was observed in the following networks: food webs (marine, estuarine, terrestrial), plant-pollinator, plantherbivores-parasitoids in the forest [119-121]. However, some authors suggest that the connectance decreases when specialists are lost or generalists are gained [122,123]. In the quill mites-doves network, the proportion of specialized species is higher compared with the bat-fly network. Additionally, some analyses focusing on the conservation and protection of biodiversity suggest that the high $\mathrm{C}$-value characterizes more stable communities, while low C-value can be an indicator of an ecological threat [122]. We hypothesize that the high C-value is observed in the stable and old hosts-parasites systems.

The Columbiformes and syringophilid mites have a long, common history. Quill mites have probably been associated with birds hosts for a very long time. Some studies based on the phylogeny of Syringophilidae and birds indicate that the quill mites of the family Syringophilidae could be associated with Neornithes birds around 66 million years ago or earlier [124]. Currently, the family Syringophilidae comprises about 400 species associated with birds from 27 orders. Most infested bird species belong to the clade Neoaves. However, quill mites species have also been found in Paleognathae (2 quill mite species), as well as Galloanserae (23 quill mite species) [20]. Considering the richness of parasites that inhabit modern birds, [124] suggested that their origins are not later than the Late Jurassic. Phylogeny analysis conducted by Skoracki et al. [78] showed that the mites on the earliest derivate branches are associated with birds of the advanced clade Neoaves. In contrast, genera associated with the earliest clades of extant birds, such as Tinamiformes (Palaeognathae) and Galloanserae (Anseriformes and Galliformes), are mosaically distributed in the core of the tree. On the other hand, ancestors of the quill mites could be associated with bird-like creatures before the K-Pg extinction event. Phylogeny analysis of parasitic mites from the superfamily Cheyletoidea (Acariformes: Prostigmata) showed that the Syringophilidae probably originated from a common ancestor with Cheyletidae, a predatory ancestor and inhabiting the litter of bird nests [124,125].

However, comparing the presented results with another host-parasites network is still unsatisfactory. The network-thinking approach used for the study of ectoparasites-hosts systems is limited. The most available research on bipartite networks was conducted on the mutualistic plant-pollinator food web. Moreover, we suggest that co-evolutionary analysis 
will be important to understand better the nature of the relationship between quill mites and doves.

\section{Conclusions}

The relation and interactions between host and parasites are still not well understood. We believe that this study focused on host specificity, prevalence, networks and evolutionary aspect has a particular role to identify the relation between host and parasites. The results of the presented study show that the quill mites belonging to family Syringophlidae and associated with pigeons and doves (Columbiformes) form stable and non-random communities.

The quill mites-doves bipartite has been characterized by a high value of nestedness, connectance, modularity, and $\mathrm{H}^{\prime}$. We suggest that the observed network architecture in this study as well as high specificity and worldwide distribution of syringophlid mites is characteristic for: high host specificity systems with a long and common history.

Supplementary Materials: The following are available online at https:/ /www.mdpi.com/article/10 $.3390 /$ ani11123392/s1, Table S1. The degree of Syringophilidae examination for each Columbiformes subfamily; Figure S1. A network matrix; Figure S2. Characters; Figure S3. Data matrix.

Author Contributions: Conceptualization, K.K.-G.; methodology, K.K.-G., J.Z.K. and M.S.; investigation, K.K.-G., J.Z.K. and M.S.; data curation, K.K.-G.; formal analysis, K.K.-G., J.Z.K. and M.S.; writing—original draft preparation, K.K.-G.; writing—review and editing, K.K.-G., M.S., M.H. and J.Z.K., supervision, M.S.; project administration, K.K.-G.; funding acquisition, M.S., M.H. and K.K.-G. All authors have read and agreed to the published version of the manuscript.

Funding: This research was funded by Slovak Research and Development Agency under the contract No. APVV-16-0411, VEGA 1/0876/21 and POWR.03.02.00-00-I006/17. Additionally, M.S. was supported by SAIA, n.o. (Slovak Academic Information Agency; 2021).

Institutional Review Board Statement: Not applicable.

Informed Consent Statement: Not applicable.

Data Availability Statement: Data is available upon request from the corresponding authors.

Acknowledgments: The authors would like to acknowledge Gerhard Haszprunar and Markus Unsoeld (Ornithological Section, Bavarian State Collection of Zoology, Munich, Germany) for making available the samples of dry bird skins for the present study.

Conflicts of Interest: The authors declare no conflict of interests. The funders had no role in the design of the study; in the collection, analyses, or interpretation of data; in the writing of the manuscript, or in the decision to publish the results.

\section{References}

1. Mora, C.; Tittensor, D.P.; Adl, S.; Simpson, A.G.B.; Worm, B. How many species are there on Earth and in the ocean? PLoS Biol. 2011, 9, e1001127. [CrossRef]

2. Okamura, B.; Hartigan, A.; Naldoni, J. Extensive uncharted biodiversity: The parasite dimension. Integr. Comp. Biol. 2018, 58 , 1132-1145. [CrossRef]

3. Dobson, A.; Lafferty, K.D.; Kuris, A.M.; Hechinger, R.F.; Jetz, W. Homage to Linneaus: How many parasites? How many hosts? Proc. Natl. Acad. Sci. USA 2008, 105, 11482-11489. [CrossRef]

4. Thomas, F.; Bonsall, M.B.; Dobson, A.P. Parasitism, biodiversity, and conservation. In Parasitism and Ecosystems; Thomas, F., Renaud, F., Guegan, J.F., Eds.; Oxford Scholarship: Oxford, UK, 2005; pp. 124-139.

5. Poulin, R. Parasite biodiversity revisited: Frontiers and constraints. Int. J. Parasitol. 2014, 44, 581-589. [CrossRef]

6. Costello, M.J. Parasite rates of discovery, global species richness and host specificity. Integr. Comp. Biol. 2016, 56, 588-599. [CrossRef] [PubMed]

7. Kissling, W.D.; Schleuning, M. Multispecies interactions across trophic levels at macroscales: Retrospective and future directions. Ecography 2015, 38, 346-357. [CrossRef]

8. Poulin, R. Network analysis shining light on parasite ecology and diversity. Trends Parasitol. 2010, 26, 492-498. [CrossRef]

9. Araújo, W.S.; Kollár, J. First characterization of a highly specialized ecological network composed by gall-inducing mites and their host plants. Int. J. Acarol. 2019, 45, 223-226. [CrossRef] 
10. Clements, J.F.; Schulenberg, T.S.; Iliff, M.J.; Roberson, D.; Fredericks, T.A.; Sullivan, B.L.; Wood, C.L. The eBird/Clements Checklist of Birds of the World. 2019. Available online: http:/ / www.birds.cornell.edu/clementschecklist/download/v2019 (accessed on 15 September 2021).

11. Gibbs, D.; Barnes, E.; Cox, J. Pigeons and Doves: A Guide to the Pigeons and Doves of the World; Yale University Press: New Haven, CT, USA, 2001; p. 615.

12. Pereira, S.L.; Johnson, K.P.; Clayton, D.H.; Baker, A.J. Mitochondrial and nuclear DNA sequences support a Cretaceous origin of Columbiformes and a dispersal-driven radiation in the Paleocene. Syst. Biol. 2007, 56, 656-672. [CrossRef] [PubMed]

13. Zachos, J.; Pagani, M.; Sloan, L.; Thomas, E.; Billups, K. Trends, rhythms, and aberrations in global climate 65 Ma to present. Science 2001, 292, 686-693. [CrossRef] [PubMed]

14. Bohaty, S.M.; Zachos, J.C.; Delaney, M.L. Foraminiferal Mg/Ca evidence for southern ocean cooling across the eocene-oligocene transition. Earth Planet Sci. Lett. 2012, 317, 251-261. [CrossRef]

15. Soares, A.; Novak, B.; James, H.; Heupink, T.; Fjeldså, J.; Gilbert, T.; Poinar, H.; Church, G.; Shapiro, B. Complete mitochondrial genomes of living and extinct pigeons revise the timing of the columbiform radiation. Evol. Biol. 2016, 16, 230. [CrossRef]

16. Kethley, J.B. A revision of the family Syringophilidae (Prostigmata: Acarina). Contrib. Am. Entomol. Inst. 1970, 5, 1-76.

17. Kethley, J.B. Population regulation in quill mites (Acari: Syringophilidae). Ecology 1971, 52, 1113-1118. [CrossRef]

18. Casto, S.D. Quill wall thickness and feeding of Syringophiloidus minor (Berlese) (Acarina: Syringophilidae). Ann. Entomol. Soc. Am. 1974, 67, 824. [CrossRef]

19. Filimonova, S.A.; Mironov, S.V. Functional morphology of the gnathosoma in the quill mite Syringophilopsis fringilla Fritsch (Acari: Prostigmata: Syringophilidae). Zoologischer Anzeiger-A J. Comp. Zool. 2010, 249, 165-180. [CrossRef]

20. Zmudzinski, M.; Skoracki, M.; Sikora, B. An Updated Checklist of Quill Mites of the Family Syringophilidae (Acariformes: Prostigmata). 2020. Available online: https://sites.google.com/site/syringophilidae/v2020 (accessed on 2 November 2021).

21. Johnston, D.E.; Kethley, J.B. A numerical phenetic study of the quill mites of the family Syringophilidae (Acari). J. Parasitol. 1973, 59, 520-530. [CrossRef]

22. Skoracki, M. Quill mites (Acari: Syringophilidae) of the Palaearctic region. Zootaxa 2011, 2840, 1-414. [CrossRef]

23. Skoracki, M.; Unsoeld, M.; Marciniak, N.; Sikora, B. Diversity of quill mites of the family Syringophilidae (Acari: Prostigmata) parasitizing owls (Aves: Strigiformes) with remarks on the host-parasite relationships. J. Med. Entomol. 2016, 53, 815-826. [CrossRef]

24. Skoracki, M.; Hromada, M.; Zmudzinski, M.; Unsoeld, M.; Sikora, B. Parasitic quill mites of the family Syringophilidae (Acariformes: Prostigmata) associated with Sub Saharan Sunbirds (Passeriformes: Nectariniidae): Species composition and host-parasite relationships. J. Med. Entomol. 2018, 55, 1464-1477. [CrossRef] [PubMed]

25. Skoracki, M.; Hromada, M.; Prevuznakova, P.; Wamiti, W. Mites of the family Syringophilidae (Acariformes: Cheyletoidea) parasitizing waxbills of the genus Estrilda (Passeriformes: Estrildidae). Syst. Appl. Acarol. 2019, 24, 1799-1808.

26. Kaszewska, K.; Skoracki, M.; Hromada, M. A review of the quill mites of the genus Gunabopicobia Skoracki and Hromada (Acariformes: Prostigmata: Syringophilidae) associated with birds of the order Columbiformes. Int. J. Acarol. 2018, 44, 288-299. [CrossRef]

27. Hirst, S. Notes on Acari parasitic on birds with descriptions of two new species. Ann. Mag. Nat. Hist. 1920, 6, 121-122. [CrossRef]

28. Clark, G.M. The acarine genus Syringophilus in North American birds. Acarologia 1964, 6, 76-92.

29. Lawrence, R.F. New mite parasites of African birds. Parasitology 1959, 49, 425-427. [CrossRef]

30. Casto, S.D. A new quill mite (Acarina: Syringophilidae) from the ground dove. Southwest. Entomol. 1980, 5, 1-5.

31. Casto, S.D. A new syringophilid mite from the white-winged dove. Tex. J. Sci. 1979, 31, 225-229.

32. Bochkov, A.V.; Mironov, S.V. Quill mites of the family Syringophilidae Lavoipierre, 1953 (Acariformes: Prostigmata) parasitic on birds (Aves) of the fauna of the former USSR. Acarina 1998, 6, 3-16.

33. Fain, A.; Bochkov, A.V.; Mironov, S.V. New genera and species of quill mites of the family Syringophilidae (Acari: Prostigmata). Bulletin de l'Institut Royal des Sciences Naturelles de Belgique 2000, 70, 33-70.

34. Bochkov, A.V.; Perez, T.M. New quill mites of the family Syringophilidae (Acari: Cheyletoidea) parasitizing Mexican parrots. Belg. J. Entomol. 2002, 4, 145-159.

35. Bochkov, A.V.; Fain, A. New and little known species of the family Syringophilidae (Acari: Cheyletoidea) from parrots (Aves: Psittaciformes). Acarina 2003, 11, 37-44.

36. Bochkov, A.V.; Williams, G.; Proctor, H. First record of Picobia zumpti (Acari: Syringophilidae) from quills of the rock pigeon in North America and description of the male. Belg. J. Entomol. 2005, 7, 121-127.

37. Skoracki, M.; Glowska, E. Quill mites (Acari: Syringophilidae) associated with columbiform birds. Genus 2008, 19, 151-160.

38. Nattress, B.; Skoracki, M. A new species and further records of quill mites (Acari: Cheyletoidea: Syringophilidae) parasitic on birds (Aves) in England. Zootaxa 2009, 2133, 49-54. [CrossRef]

39. Glowska, E.; Skoracki, M. Meitingsunes, a new genus of the ectoparasitic quill mites (Acari: Cheyletoidea: Syringophilidae). Zootaxa 2010, 2514, 61-67. [CrossRef]

40. Skoracki, M.; Dabert, J. A review of parasitic mites of the family Syringophilidae (Acari, Prostigmata) from African birds, with descriptions of four new species. Acta Parasitol. 2002, 47, 137-146.

41. Skoracki, M.; Hromada, M. A review of picobiine mites (Acari: Syringophilidae: Picobiinae) parasitising African birds. Folia Parasitol. 2013, 60, 192-212. [CrossRef] 
42. Kaszewska, K.; Skoracki, M. Two new quill mite species of the genus Psittaciphilus Fain, Bochkov \& Mironov, 2000 (Acariformes: Syringophilidae) associated with pigeons and doves (Columbiformes: Columbidae). Syst. Parasitol. 2018, 95, 953-958. [PubMed]

43. Kaszewska, K.; Kavetska, K.; Skoracki, M. Two new species of quill mites of the family Syringophilidae (Acariformes: Cheyletoidea) associated with treronine doves (Columbiformes: Columbidae: Treroninae). Zootaxa 2014, 3846, 293-300. [CrossRef]

44. Kaszewska, K.; Skoracki, M.; Kavetska, K. Two new Meitingsunes species (Acari: Syringophilidae) from Indonesian doves (Columbiformes: Columbidae). Zootaxa 2016, 4109, 479-486. [CrossRef] [PubMed]

45. Kaszewska, K.; Skoracki, M.; Hromada, M. The mites of the genus Meitingsunes Glowska and Skoracki (Acariformes: Syringophilidae) associated with pigeons and doves (Aves: Columbiformes): Taxonomic studies with description of two new species. Int. J. Acarol. 2020, 46, 439-455. [CrossRef]

46. Kaszewska, K.; Skoracki, M.; Kosicki, Z.J.; Hromada, M. New species and records of the quill mites of the genus Peristerophila Kethley, 1970 (Acariformes: Syringophilidae) associated with pigeons and doves (Aves: Columbiformes). Zootaxa 2020, 4878, 349-361. [CrossRef]

47. Skoracki, M.; Sikora, B.; Spicer, G.S. A review of the subfamily Picobiinae Johnston and Kethley, 1973 (Acariformes: Prostigmata: Syringophilidae). Zootaxa 2016, 4113, 1-95. [CrossRef] [PubMed]

48. Skoracki, M.; Lontkowski, J.; Stawarczyk, T. New taxa of the parasitic quill mites associated with accipitrid birds indicating close relationship of falconid birds to Psittaci-Columbi clade. J. Nat. Hist. 2010, 44, 1203-1214. [CrossRef]

49. Skoracki, M.; Hromada, M.; Kaszewska, K.; Sikora, B. Females of the quill mite genera Peristerophila and Castosyringophilus (Acariformes: Syringophilidae) are two morphological forms: Ontogenetic and population evidences. Syst. Appl. Acarol. 2020, 25, 1803-1820.

50. Skoracki, M.; Kaszewska, K.; Unsoeld, M.; Skorupski, M. First record of parasitic quill mites of the family Syringophilidae (Acari: Prostigmata: Cheyletoidea) on an avian representative of the order Caprimulgiformes. Int. J. Acarol. 2015, 41, 128-131. [CrossRef]

51. Dormann, F.; Gruber, B.; Fründ, J. Introducing the bipartite package: Analysing ecological networks. R News 2008, 8, 1-11.

52. Blüthgen, N.; Menzel, F.; Bliithgen, N. Measuring specialization in species interaction networks. BMC Ecol. 2006, 9, 6-9.

53. Blüthgen, N. Why network analysis is often disconnected from community ecology: A critique and an ecologist's guide. Basic Appl. Ecol. 2010, 11, 185-195. [CrossRef]

54. Blüthgen, N.; Fründ, J.; Vázquez, D.P.; Menzel, F. What do interaction network metrics tell us about specialization and biological traits. Ecology 2008, 89, 3387-3399. [CrossRef]

55. Atmar, W.; Patterson, B.D. The measure of order and disorder in the distribution of species in fragmented habitat. Oecologia 1993, 96, 373-382. [CrossRef] [PubMed]

56. Bascompte, J.; Jordano, P.; Melia, C.J.; Olesen, J.M. The nested assembly of plant-animal mutualistic networks. Proc. Natl. Acad. Sci. USA 2003, 100, 9383-9387. [CrossRef] [PubMed]

57. Pavlopoulos, G.A.; Kontou, P.I.; Pavlopoulou, A.; Bouyioukos, C.; Markou, E.; Bagos, P.G. Bipartite graphs in systems biology and medicine: A survey of methods and applications. GigaScience 2018, 7, giy014. [CrossRef]

58. Fortuna, M.A.; Stouffer, D.B.; Olesen, J.M.; Jordano, P.; Mouillot, D.; Krasnov, B.R.; Poulin, R.; Bascompte, J. Nestedness versus modularity in ecological networks: Two sides of the same coin? J. Anim. Ecol. 2010, 79, 811-817. [CrossRef] [PubMed]

59. Newman, M.E.J.; Barabási, A.L.; Watts, D.J. The Structure and Dynamics of Networks; Princeton University Press: Princeton, NJ, USA, 2006; p. 592.

60. Guimera, R.; Amaral, L.A.N. Functional cartography of complex metabolic networks. Nature 2005, 433, 895-900. [CrossRef] [PubMed]

61. Dormann, C.F.; Strauss, R. A method for detecting modules in quantitative bipartite networks. Methods Ecol. Evol. 2014, 5, 90-98. [CrossRef]

62. Carstensen, D.W.; Sabatino, M.; Morellato, L.P.C. Modularity, pollination systems, and interaction turnover in plant-pollinator networks across space. Ecology 2016, 97, 1298-1306. [CrossRef]

63. Reiczigel, J.; Marozzi, M.; Fábián, I.; Rózsa, L. Biostatistics for parasitologists-A primer to Quantitative Parasitology. Trends Parasitol. 2019, 35, 277-281. [CrossRef]

64. Page, R.D.M. NDE, NEXUS Data Editor 0.5.0; University of Glasgow: Glasgow, UK, 2001.

65. Nixon, K.C. Program and documentation. In WINCLADA; Version 0.9.9b; Ithaca: New York, NY, USA, 1999; Available online: https:/ / cladistics.com (accessed on 2 November 2021).

66. Strong, E.E.; Lipscomb, D. Character coding and inapplicable data. Cladistics 1999, 15, 363-371. [CrossRef]

67. Brazeau, M.D. Problematic character coding methods in morphology and their effects. Biol. J. Linn. Soc. 2011, 104, 489-498. [CrossRef]

68. Swofford, D.L. PAUP* Phylogenetic Analysis Using Parsimony ( ${ }^{*}$ and Other Methods), Version 4; Sinauer Associates: Sunderland, MA, USA, 2002.

69. Muller, K. PRAP_Computation of Bremer support for large data sets. Mol. Phyl. Evol. 2004, 31, 780-782. [CrossRef] [PubMed]

70. Page, R.D.M. TreeView: An application to display phylogenetic trees on personal computers. Comput. Appl. Biosci. 1996, 12, 357-358.

71. Jetz, W.; Thomas, G.H.; Joy, J.B.; Hartmann, K.; Mooersm, O.A. The global diversity of birds in space and time. Nature 2012, 491, 444-448. [CrossRef] [PubMed] 
72. Drummond, A.J.; Rambaut, A. BEAST: Bayesian evolutionary analysis by sampling trees. BMC Evol. Biol. 2007, 7, 214. [CrossRef] [PubMed]

73. Caira, J.N.; Jensen, K.; Holsinger, K.I. On a new index of host specificity. In Taxonomie, Écologie et Évolution des Metazoaires Parasites; Combes, C., Jourdane, J., Eds.; Presses Universitaires de Perpignan: Perpignan, France, 2003; pp. 161-201.

74. Holt, B.G.; Lessardb, J.P.; Borregaard, M.K.; Fritz, S.A.; Araújo, M.B.; Dimitrov, D.; Fabre, P.H.; Graham, C.H.; Graves, G.R.; Jønsson, K.A.; et al. An update of Wallace's zoogeographic regions of the world. Science 2013, 339, 74-78. [CrossRef] [PubMed]

75. Casto, S.D. Host records and observations of quill mites (Acarina: Syringophilidae) from Texas birds. Southwest. Entomol. 1976, 1, 155-160.

76. Skoracki, M.; Sikora, B. New ectoparasitic mites of the family Syringophilidae (Acari: Prostigmata: Cheyletoidea) associated with birds from Argentina. Zootaxa 2002, 27, 1-8. [CrossRef]

77. Klimovičová, M.; Skoracki, M.; Wamiti, W.; Hromada, M. Quill mites of the subfamily Picobiinae (Acari: Syringophilidae) parasitising African birds, with description of two new species. Folia Parasitol. 2014, 61, 394-400. [CrossRef]

78. Skoracki, M.; Hendricks, S.A.; Spicer, G.S. Systematics of the genus Syringophilopsis Kethley, 1970 (Acari: Prostigmata: Syringophilidae) with description of three new species from North American passerines. Zootaxa 2011, 2793, 1-22. [CrossRef]

79. Hromada, M.; Klimovičová, M.; Unsöld, M.; Skoracki, M. Host-parasite relationships in the system composed by cuckoos and quill mites. Syst. Appl. Acarol. 2016, 21, 528-536.

80. Skoracki, M.; Glowska, E.; Bochkov, A.V. Phylogeny of quill mites of the family Syringophilidae (Acari: Prostigmata) based on their external morphology. Eur. J. Entomol. 2013, 110, 663-675. [CrossRef]

81. Skoracki, M.; Sikora, B. Tinamiphilopsis elegans gen. nov. et sp. nov., a first record of the quill mites (Acari, Syringophilidae) from tinamou birds (Tinamiformes, Tinamidae). Acta Parasitol. 2004, 49, 348-352.

82. Jarvis, E.D.; Mirarab, S.; Aberer, A.J.; Li, B.; Houde, P.; Li, C.; Ho, S.Y.W.; Faircloth, B.C.; Nabholz, B.; Howard, J.T.; et al. Whole-genome analyses resolve early branches in the tree of life of modern birds. Science 2014, 346, 1320-1331. [CrossRef]

83. Prum, R.O.; Jacob, S.B.; Dornburg, A.; Field, D.J.; Townsend, J.P.; Lemmon, E.M.; Lemmon, A.R. A comprehensive phylogeny of birds (Aves) using targeted next-generation DNA sequencing. Nat. Lett. 2015, 15697, 569-573. [CrossRef] [PubMed]

84. Kimball, R.T.; Oliveros, C.H.; Wang, N.; White, N.D.; Barker, F.K.; Field, D.J.; Ksepka, D.T.; Chesser, R.T.; Moyle, R.G.; Braun, M.J.; et al. A phylogenomic super tree of birds. Diversity 2019, 11, 109. [CrossRef]

85. Skoracki, M.; Hromada, M.; Sikora, B. Castosyringophilus meropis sp. n. (Acariformes: Syringophilidae)—A new quill mite species parasitising the world population of Merops apiaster Linnaeus (Coraciiformes: Meropidae). Folia Parasitol. 2017, 64, 24. [CrossRef]

86. Marciniak, N.; Skoracki, M.; Hromada, M. Peristerophila nestoriae, a new species of quill mite of the family Syringophilidae (Acariformes: Prostigmata) parasitizing New Zealand Kaka Nestor meridionalis (Gmelin) (Psittaciformes: Strigopidae). N. Z. J. Zool. 2019, 46, 348-352. [CrossRef]

87. Rebrassier, R.E.; Martin, E.D. Syringophilus bipectinatus a quill mite of poultry. Science 1932, 76, 128. [CrossRef] [PubMed]

88. Casto, S.D. The effect of the postjuvenal molt in the House Sparrow on infestations of the quill mite, Syringophiloidus minor (Berlese) (Acarina: Syringophilidae). J. Med. Entomol. 1975, 12, 23-27. [CrossRef] [PubMed]

89. Skoracki, M.; Hromada, M.; Tryjanowski, P. Description of a new species of quill mite Syringophiloidus weiszii sp. n. (Acari, Prostigmata, Syringophilidae) from Great Grey Shrike Lanius excubitor. Acta Parasitol. 2001, 46, 30-34.

90. Skoracki, M.; Møller, A.P.; Tryjanowski, P. A new species of parasitic mites of the genus Syringophiloidus Kethley, 1970 (Acari: Syringophilidae) from the Barn Swallow Hirundo rustica Linnaeus, 1758. Parasite 2003, 10, 17-20. [CrossRef]

91. Skoracki, M.; Michalik, J.; Sikora, B. Prevalence and habitat preference of quill mites (Acari, Syringophilidae) parasitizing forest passerine birds in Poland. Acta Parasitol. 2010, 55, 188-193. [CrossRef]

92. Hache, S.; Bayne, E.M.; Villard, M.A.; Proctor, H.C.; Davis, C.S.; Stralberg, D.; Janes, J.K.; Hallworth, M.T.; Foster, K.R.; Chidambara-Vasi, E.; et al. Phylogeography of a migratory songbird across its Canadian breeding range: Implications for conservation units. Ecol. Evol. 2017, 7, 6078-6088. [CrossRef]

93. Grossi, A.; Proctor, H. The distribution of quill mites (Betasyringophiloidus seiuri) among flight feathers of the ovenbird (Seiurus aurocapilla). J. Parasitol. 2020, 106, 82-89. [CrossRef]

94. Pires, E.O.; Daemon, E. Biological and ecological aspects of quill mites, parasites of domestic hen Gallus gallus domesticus (Aves, Phasianidae) from rusting breeding locations in the municipality of Juiz de Fora, Minas Gerais, Brasil. Revista Brasileira de Zoociencias 2007, 9, 95-102.

95. Skirnisson, K.; Nielsen, Ó.K. Quill mite infestation of rock ptarmigan Lagopus muta (Aves: Phasianidae) in relation to year and host age, sex, body condition, and density. Parasitol. Res. 2019, 118, 2643-2650. [CrossRef]

96. Skirnisson, K.; Palsdottir, G.R. Past and present status of poultry parasites in Iceland. Icel. Agric. Sci. 2020, 33, 3-14. [CrossRef]

97. Jardim, C.C.; Cunha, L.M.; Do Carmo Rezende, L.; Teixeira, C.M.; Silva Martins, N.R.; Oliveira, P.R.; Leite, R.C.; Faccini, J.L.H.; Leite, R.C. Quill mites in Brazilian psittacine birds (Aves: Psittaciformes). J. Zoo Wildl. Med. 2012, 43, 511-516. [CrossRef]

98. Doster, G.L.; Wilson, N.; Kellogg, F.E. Ectoparasites collected from bobwhite quail in the south-eastern United States. J. Wildl. Dis. 1980, 5, 515-520. [CrossRef]

99. Órdenes, J.S.M.; Ibáñez, C.B.; Contreras, L.R.; Schmäschke, R.; Daugschies, A.; González-Acuña, D. Ectoparasitism in the common chimango caracara Milvago chimango chimango (Vieillot, 1816) (Aves, Falconidae) in the Nuble Area, Chile. Lundiana 2005, 6, 49-55.

100. Goulart, T.M.; Moraes, D.L.; Prado, A.P. Mites associated with the eared dove, Zenaida auriculata (Des Murs, 1847), in São Paulo State, Brazil. Zoosymposia 2011, 6, 267-274. [CrossRef] 
101. Moraes, D.L.; Goulart, T.M.; Prado, A.P. Mites associated with the ruddy ground dove, Columbina talpacoti (Temminck, 1810), in São Paulo State, Brazil. Zoosymposia 2011, 6, 275-281. [CrossRef]

102. Gritsenko, E.F. The biology and ecology of the quill mite Syringophilus bipectinatus Heller, 1880. In Proceedings of the 3rd International Congress of Acarology, Prague, Czechoslovakia, 31 August-6 September 1971; Milan, D., Rosicky, B., Eds.; Springer: Dordrecht, The Netherlands, 1973; pp. 515-516.

103. Skoracki, M.; Kosicki, J.Z.; Kwieciński, Z. Distribution of the parasitic mite Bubophilus aegolius sp. n. (Acariformes: Syringophilidae) on the Boreal Owl Aegolius funereus (L) (Strigiformes: Strigidae) and the low effectiveness of infestation. Eur. Zool. J. 2021, 88, 352-362. [CrossRef]

104. Schmäschke, R.; Sachse, M.; Eulenberger, K.; Schöne, R. Quill Mites_Little Known Parasites of Birds; Internationalen Symposiums über die Erkrankungen der Zoo und Wildtiere: Berlin, Germany, 2003; Volume 41, pp. 127-133.

105. MacArthur, R.H.; Levins, R. The limiting similarity, convergence and divergence of coexisting species. Am. Nat. 1967, 101, 377-385. [CrossRef]

106. Amarasekare, P. Competitive coexistence in spatially structure environments: A synthesis. Ecol. Lett. 2003, 6, 1109-1122. [CrossRef]

107. Gonzalez-Acuna, D.; Venzal, J.M.; Keirans, J.E.; Robbins, R.G.; Ippi, S.; Guglielmone, A.A. New host and locality records for the Ixodes auritulus (Acari: Ixodidae) species group, with a review of host relationships and distribution in the Neatropical Zoogeographic Region. Exp. Appl. Acarol. 2005, 37, 147-156. [CrossRef]

108. Huang, L.Q.; Guo, X.-G.; Wu, D.; Zhou, D.H. Distribution and ecological niches of gamasid mites (Acari: Mesostigmata) on small mammals in Southwest China. Psyche 2010, 934508, 934508. [CrossRef]

109. Bochkov, A.V.; Labrzycka, A.; Skoracki, M.; Saveljev, A.P. Fur mites of the genus Schizocarpus Trouessart (Acari: Chirodiscidae) parasitizing the Eurasian beaver Castor fiber belorussicus Lavrov (Rodentia: Castoridae) in NE Poland (Suwałki). Zootaxa 2012, 3162, 39-59. [CrossRef]

110. Stefan, L.M.; Gómez-Díaz, E.; Elguero, E.; Proctor, H.C.; McCoy, K.D.; González-Solís, J. Niche partitioning of feather mites within a seabird host, Calonectris borealis. PLoS ONE 2015, 10, e0144728. [CrossRef]

111. Glowska, E.; Dragun-Damian, A.; Dabert, J. DNA-barcoding contradicts morphology in quill mite species Torotrogla merulae and T. rubeculi (Prostigmata: Syringophilidae). Folia Parasitol. 2013, 60, 51-60. [CrossRef]

112. Landi, P.; Minoarivelo, H.O.; Brännström, A.; Hui, C.; Dieckmann, U. Complexity and stability of ecological networks: A review of the theory. Popul. Ecol. 2018, 60, 319-345. [CrossRef]

113. Durán, A.; Saldaña-Vázquez, R.; Graciolli, G.; Peinado, L. Specialization and Modularity of a Bat Fly Antagonistic Ecological Network in a Dry Tropical Forest in Northern Colombia. Acta Chiropterologica 2019, 20, 503-510. [CrossRef]

114. Lewis, O.T.; Memmott, J.; Lasalle, J.; Lyal, C.; Whitefoord, C.; Godfray, C.J. Blackwell Science, Ltd. Structure of a diverse tropical forest insect-Parasitoid community. J. Anim. Ecol. 2002, 71, 855-873. [CrossRef]

115. Dormann, C.F.; Fründ, J.; Blüthgen, N.; Gruber, B. Indices, graphs and null models: Analysing bipartite ecological networks. Open Ecol. J. 2009, 2, 7-24. [CrossRef]

116. Thebault, E.; Fontaine, C. Stability of ecological communities and the architecture of mutualistic and trophic networks. Science 2010, 329, 853-856. [CrossRef] [PubMed]

117. Olesen, J.M.; Bascompte, J.; Dupont, Y.L.; Jordano, P. The modularity of pollination networks. Proc. Natl. Acad. Sci. USA 2007, 104, 19891-19896. [CrossRef]

118. Delmas, E.; Besson, M.; Brice, M.H.; Burkle, L.A.; Dalla Riva, G.V.; Fortin, M.J.; Gravel, D.; Guimarães, P.R., Jr.; Hembry, D.H.; Newman, E.A.; et al. Analysing ecological networks of species interactions. Biol. Rev. 2018, 94, 16-36. [CrossRef]

119. Briand, F. Environmental control of food web structure. Ecology 1983, 64, 253-263. [CrossRef]

120. Memmott, J.; Waser, N.M. Integration of alien plants into a native flower pollinator visitation web. Proc. R. Soc. Lond. Ser. B-Biol. Sci. 2002, 269, 2395-2399. [CrossRef]

121. Heleno, R.H.; Lacerda, I.; Ramos, J.A.; Memmott, J. Evaluation of restoration effectiveness: Community response to the removal of alien plants. Ecol. Appl. 2010, 20, 1191-1203. [CrossRef]

122. Devictor, V.; Julliard, R.; Jig, F. Distribution of specialist and generalist species along spatial gradients of habitat disturbance and fragmentation. Oikos 2008, 117, 507-514. [CrossRef]

123. Heleno, R.; Devoto, M.; Pocock, M. Connectance of species interaction networks and conservation value: Is it any good to be well connected? Ecol. Indic. 2012, 14, 7-10. [CrossRef]

124. Bochkov, A.V. The classification and phylogeny of the mite superfamily Cheyletoidea (Acari, Prostigmata). Entomol. Rev. 2002, 82, 643-664.

125. Volgin, V.I. Kleschi Semeistva Cheyletidae Mirovoi Fauny; Akademiia NAUK SSSR Zoologicheskii Institut: Leningrad, Russia, 1969; Volume 101, p. 432. 